Publ. RIMS, Kyoto Univ.

17 (1981), 25-63

\title{
The Theory of Vector Valued Fourier Hyperfunctions of Mixed Type. I
}

By

\author{
Shigeaki NAGAMACHI*
}

\begin{abstract}
The soft resolution $\left(\mathscr{I}_{(0, p)}, \bar{\partial}\right)$ of the sheaf $\widetilde{\mathcal{O}}_{k, l}$ of slowly increasing holomorphic functions of $(k, l)$ type is constructed so that the section modules $\mathscr{F}_{(0, p)}(\Omega)$ are Fréchet nuclear spaces. Using the above resolution, we construct the mixed type Fourier hyperfunctions which take their values in Fréchet spaces.
\end{abstract}

\section{§ 0. Introduction}

Recently the theory of Fourier hyperfunctions which take their values in a Hilbert space was developed by Y. Ito and S. Nagamachi [5], and it was applied to the formulation of the axiomatic quantum field theory by S. Nagamachi and N. Mugibayashi [9]. On the other hand P.D. F. Ion and T. Kawai [4] developed the theory of hyperfunctions which take their values not in a Hilbert space but in a Fréchet space.

There exist another kind of Fourier hyperfunctions which were announced in T. Kawai [6] to be published as modified Fourier hyperfunctions but actually was not published. It turned out that the new type Fourier hyperfunctions are useful in order to give the equivalent Euclidean formulation of the quantum field theory. We call this new Fourier hyperfunction the second type Fourier hyperfunction in distinction from the old one, the first type Fourier hyperfunction which was developed in T. Kawai [6]. In S. Nagamachi and N. Mugibayashi [10], the mixed type Fourier hyperfunctions were used for above purpose. The mixed type Fourier hyperfunctions are those Fourier hyperfunctions which are of the first type in some variables and of the second type in

Communicated by M. Sato, October 27, 1978.

* Department of Mathematics, Faculty of Engineering, Tokushima University, Tokushima 770 , Japan. 
other variables. The space of mixed type Fourier hyperfunctions is a convenient subspace of the space of the second type Fourier hyperfunctions. Recently, Y. Saburi [13], [14] completed the theory of the second type Fourier hyperfunctions which he called modified Fourier hyperfunctions there, whereas in S. Nagamachi and N. Mugibayashi [11], the theory is developed only to fulfill the minimal needs for the application of the quantum field theory.

In this paper we construct the theory of mixed type Fourier hyperfunctions which take their values in a Fréchet space. Since the unbounded operator algebra, as was developed by A. Inoue [3], has a Fréchet space structure, it will be possible to define operator valued Fourier hyperfunctions and apply them to the quantum field theory.

In Section 2, we define (Definition 2.13) the space $\mathscr{E}(\Omega)$ of slowly increasing $C^{\infty}$-functions, and prove (Theorem 2.19 ) that $\mathscr{F}(\Omega)$ is a Fréchet nuclear space.

In Section 4, we prove the first main result, Theorem 4. 11, which says that the sheaf $\widetilde{\mathcal{O}}_{k, l}$ of the slowly increasing holomorphic functions has a soft resolution,

$$
0 \rightarrow \widetilde{\mathcal{O}}_{k, l} \rightarrow \mathscr{I}_{(0,0)} \stackrel{\bar{\partial}}{\rightarrow} \cdots \stackrel{\bar{\partial}}{\rightarrow} \mathscr{I}_{(0, n)} \rightarrow 0,
$$

where $\mathscr{I}_{(0, p)}$ are sheaves of slowly increasing $C^{\infty}-(0, p)$ form.

In Section 5, we prove the vanishing theorems of cohomology groups whose coefficients are in a sheaf $\widetilde{\mathcal{O}}_{k, l}$, in a way similar to T. Kawai [6] and Y. Saburi [13], [14].

In Section 6, using the resolution of $\widetilde{\mathcal{O}}_{k, l}$ (Theorem 4.11), we transfer the results in Section 5 (scalar valued case) to the vector valued case by the tensoring method used in P.D. F. Ion and T. Kawai [4], where it is important that $E$ is a Fréchet space and $\mathscr{I}(\Omega)$ is a Fréchet nuclear space. Thus we have the second main result, Definition 6. 9 and Theorem 6. 11, where the space ${ }^{E} \mathscr{R}_{k, l}(\Omega)$ of mixed type $E$-valued Fourier hyperfunctions is defined and the flabbiness of the sheaf is proved.

\section{$\S 1$. Notations and Conventions}

Let $z \in \boldsymbol{C}^{n}, z=x+i y$ and $x \in \boldsymbol{R}^{n}, y \in \boldsymbol{R}^{n}$. We follow the standard 
notations of $n$-tuple of numbers. Thus, let $\alpha=\left(\alpha_{1}, \cdots, \alpha_{n}\right)$ be $n$-tuple of nonnegative integers, then $D^{\alpha}=\partial^{|\alpha|} / \partial x_{1}^{\alpha_{1}} \cdots \partial x_{n}^{\alpha_{n}}$, where $|\alpha|=\alpha_{1}+\cdots+\alpha_{n}$. The notation $|z|^{p}$ is used to denote $|z|^{p}=\left|z_{1}\right|^{p}+\cdots+\left|z_{n}\right|^{p}$, in particular $|z|=\left|z_{1}\right|+\cdots+\left|z_{n}\right| . \quad d \lambda$ is the Lebesgue measure on $\boldsymbol{R}^{2 n} ; d \lambda=d x d y$ $=d x_{1} \cdots d x_{n} d y_{1} \cdots d y_{n}$.

If we consider $\boldsymbol{C}^{n}$ to be the product $\prod_{i=1}^{j}\left(\boldsymbol{C}^{k_{i}} \times \boldsymbol{C}^{t_{i}}\right)$, where $n=\sum_{i=1}^{j} n_{i}$ and $n_{i}=k_{i}+l_{i}$, we sometimes use the notation that $z=\left(z_{1}^{\prime}, \cdots, z_{j}^{\prime}\right) \in C^{n}$ where $z_{i}^{\prime}=\left(\underline{z}_{k_{i}}, \underline{z}_{l_{i}}\right) \in C^{n_{i}}$ and $\underline{z}_{k_{i}}=\left(z_{1}, \cdots, z_{k_{i}}\right) \in C^{k_{i}}, \underline{z}_{l_{i}}=\left(z_{1}, \cdots, z_{l_{i}}\right) \in C^{l_{i}}$.

For the function $f(z)$ defined in $C^{n}$, the notation $D^{\alpha} f(x, y)$ or simply $D^{\alpha} f(z)$ is used to denote $\partial^{|\alpha|} f(x, y) / \partial x_{1}^{\alpha_{1}} \ldots \partial x_{n}^{\alpha_{n}} \partial y_{1}^{\alpha_{n+1}} \ldots \partial y_{n}^{\alpha_{2 n}}$ for $2 n$-tuple of nonnegative integers $\alpha=\left(\alpha_{1}, \cdots, \alpha_{2 n}\right)$.

\section{§ 2. Function Spaces}

In this section, we introduce various function spaces which are used in the theory of Fourier hyperfunctions. To describe the order of the growth or decay at infinity, it is convenient to introduce points at infinity and the neighbourhoods of the points at infinity.

Definition 2. 1 (T. Kawai [6]). We denote by $\boldsymbol{D}^{n}$ the compactification $\boldsymbol{R}^{n} \cup \boldsymbol{S}_{\infty}^{n-1}$ of $\boldsymbol{R}^{n}$, where $\boldsymbol{S}_{\infty}^{n-1}$ is the $(n-1)$-dimensional sphere at infinity. To each $x \in \boldsymbol{R}^{n}-\{0\}$, we associate a point $x_{\infty}$ on $\boldsymbol{S}_{\infty}^{n-1}$ by identifying $\boldsymbol{S}_{\infty}^{n-1}$ with $\left[\boldsymbol{R}^{n}-\{0\}\right] / \boldsymbol{R}_{+}$where $\boldsymbol{R}_{+}=\{x \in \boldsymbol{R} ; x>0\}$.

A natural topology is given to the space $\boldsymbol{D}^{n}$.

(i) For $x \in \boldsymbol{R}^{n}$, a fundamental system of neighbourhoods of $x$ is the set of open balls containing the point $x$.

(ii) For $x \in \boldsymbol{S}_{\infty}^{n-1}$, a fundamental system of neighbourhoods of $x\left(=y_{\infty}\right)$ is given by $\left\{(C+a) \cup C_{\infty} ; C_{\infty} \ni y_{\infty}\right\}$, where $C$ is an open cone generated by some open neighbourhood of $y$ with its vertex at the origin, $a$ is some vector in $\boldsymbol{R}^{n}$, so that $C+a$ is a cone with its vertex at $a$, and $C_{\infty}$ signifies the points at infinity of that cone.

In the theory of Fourier hyperfunctions of the second type or mixed type, we use the following compactification.

Definition 2. 2. Let $\boldsymbol{C}^{k} \times \boldsymbol{R}^{l}, k+l=n$, be identified with $\boldsymbol{R}^{n+k}$. We 
denote its compactification by $\boldsymbol{D}^{k, l}$, i.e., $\boldsymbol{D}^{k, l}=\boldsymbol{R}^{n+k} \cup \boldsymbol{S}_{\infty}^{n+k-1}$. The topology of $\boldsymbol{D}^{k, l}$ is given by the same way as $\boldsymbol{D}^{n}$ in Definition 2.1.

It is evident that the closure of $\boldsymbol{R}^{n}=\boldsymbol{R}^{k+l}$ in $\boldsymbol{D}^{k, l}$ is identical with $\boldsymbol{D}^{n}$. We write $\boldsymbol{Q}^{k, l}=\boldsymbol{D}^{k, l} \times i \boldsymbol{R}^{l}$ and for multiindices $k=\left(k_{1}, \cdots, k_{j}\right)$, $l=\left(l_{1}, \cdots, l_{j}\right)$, we define $\boldsymbol{Q}^{k, l}=\prod_{i=1}^{j} \boldsymbol{Q}^{k_{i}, l_{i}}$ equipped with the product topology.

Now we define the sheaves which are very important in the theory of Fourier hyperfunctions.

Definition 2. 3. (The sheaf of slowly increasing holomorphic functions.) Let $\Omega$ be an open set in $\boldsymbol{Q}^{k, l}$ (where $k, l$ are nonnegative integers or multiindices). We denote by $\widetilde{\mathcal{O}}_{k, l}$ the sheaf determined by a presheaf $\left\{\widetilde{\mathcal{O}}_{k, l}(\Omega)\right\}$, where $\widetilde{\mathcal{O}}_{k, l}(\Omega)$ is the set of all holomorphic functions $f(z)$ $\left(\in \mathcal{O}\left(\Omega \cap \boldsymbol{C}^{n}\right), n=|k|+|l|\right)$ such that $\sup _{\boldsymbol{z} \in K_{\cap} \boldsymbol{C}^{n}}|f(z)| e^{-\varepsilon|z|}<\infty$ for any $\varepsilon>0$ and any compact set $K$ in $\Omega$.

Definition 2. 4. (The sheaf of rapidly decreasing holomorphic functions.) We denote by $\mathcal{Q}_{k, l}$ the sheaf determined by a presheaf $\left\{\mathcal{O}_{k, l}(\Omega)\right\}$, where $\Omega$ is an open set in $Q^{k, l}$ and $\mathcal{Q}_{k, l}(\Omega)$ is the set of all holomorphic functions $f(z)\left(\in \mathcal{O}\left(\Omega \cap C^{n}\right)\right)$ such that for any compact set $K$ in $\Omega$ there exists some positive constant $\delta_{K}$ and the estimate $\sup _{z \in K \cap \boldsymbol{C}^{n}}|f(z)| e^{\delta_{K}|z|}<\infty$ holds.

Definition 2. 5. (Topology of $\mathcal{Q}_{k, l}(K)$.) Let $K$ be a compact set in $\boldsymbol{Q}^{k, l}$. We give the space $\mathcal{Q}_{k, l}(K) \quad\left(=\Gamma\left(K, \mathcal{O}_{k, l}\right)\right.$; the space of sections of the sheaf $\mathcal{Q}_{k, l}$ on $K$ ) the inductive limit topology $\underset{\longrightarrow}{\lim } \mathcal{O}_{c}^{m}\left(V_{m}\right)$, where $\left\{V_{m}\right\}$ is a fundamental system of neighbourhoods of $\vec{K}$ in $\boldsymbol{Q}^{k, l}$, satisfying $V_{m} \supseteq V_{m+1}$ (the closure $\bar{V}_{m+1}$ of $V_{m+1}$ is contained in the interior $\stackrel{\circ}{V}_{m}$ of $V_{m}$ with respect to the topology of $\left.\boldsymbol{Q}^{k, l}\right)$, and $\mathcal{O}_{c}^{m}\left(V_{m}\right)$ is the Banach space of all holomorphic functions $f(z)$. ( $\left.\in \mathcal{O}\left(V_{m} \cap C^{n}\right)\right)$ that are continuous in $\bar{V}_{m} \cap \boldsymbol{C}^{n}$ and for which $|f(z)| \leqq C e^{-|z| / m}$ holds for some constant $C$ (depending on $f)$. The norm of $\mathcal{O}_{c}^{m}\left(V_{m}\right)$ is defined by $\|f\|_{m}=\sup _{\boldsymbol{z} \in \bar{\nabla}_{m} \cap \boldsymbol{C}^{n}}|f(z)| e^{|z| / m}$.

Let $X$ and $Y$ be locally convex spaces. A linear mapping $u: X \rightarrow Y$ is said to be weakly compact (compact) if there is a neighbourhood $V$ 
of zero in $X$ such that $u(V)$ is relatively weakly compact (relatively compact).

Definition 2. 6 (H. Komatsu [7]). A projective (injective) sequence of locally convex spaces with (one-one) continuous linear mappings:

$$
\begin{aligned}
& X_{1} \leftarrow X_{2} \leftarrow \cdots \leftarrow X_{n} \leftarrow \cdots \\
& \left(X_{1} \rightarrow X_{2} \rightarrow \cdots \rightarrow X_{n} \rightarrow \cdots\right)
\end{aligned}
$$

is said to be weakly compact or compact if all mappings are weakly compact or compact respectively. The limit space $\underset{\lim }{\longleftarrow} X_{j}\left(\stackrel{\lim }{\longrightarrow} X_{j}\right)$ of a weakly compact or compact projective (injective) sequence is said to be $\left(F S^{*}\right)$ or $(F S)\left(\left(D F S^{*}\right)\right.$ or $\left.(D F S)\right)$ respectively.

Proposition 2. 7. Let $K$ be a compact set in $\boldsymbol{Q}^{k, l}$, then $\mathcal{Q}_{k, l}(K)$ is a DFS space.

Proof. We choose the fundamental system of neighbourhoods $\left\{V_{m}\right\}$ of $K$ so that $K$ intersects all of the connected components of $V_{m}$. Then the natural mapping from $\mathcal{O}_{c}^{m}\left(V_{m}\right)$ to $\mathcal{O}_{c}^{m+1}\left(V_{m+1}\right)$ is one to one and continuous. It suffices to show that the mapping is compact. Let $\left\{f_{n}\right\}$ be a sequence of the elements in $\mathcal{O}_{c}^{m}\left(V_{m}\right)$ which satisfy $\left\|f_{n}\right\|_{m} \leqq M$. Then we can extend $e^{|z| / m+1} f_{n}(z)$ to $\bar{V}_{m+1}$ continuously by defining $e^{|z| / m+1} f_{n}(z)$ $=0$ for $z \in \bar{V}_{m+1} \cap\left(\boldsymbol{C}^{n}\right)^{c}$, and there holds $e^{|z| / m+1} f_{n}(z) \leqq M$ (uniformly bounded). By using the Cauchy integral formula, it follows from the uniformly boundedness that, for any $\varepsilon>0$ and $z_{0} \in \bar{V}_{m+1}$, we can find a neighbourhood $U\left(z_{0}\right)$ such that, for all $f_{n}(z), z \in U\left(z_{0}\right)$ implies $\mid e^{|z| / m+1}$ $\times f_{n}(z)-e^{\left|z_{0}\right| / m+1} f_{n}\left(z_{0}\right) \mid<\varepsilon$ (equicontinuous). Since $\bar{V}_{m+1}$ is compact, Ascoli's theorem shows that there exists a subsequence $\left\{e^{|z| / m+1} f_{n_{k}}(z)\right\}$ which converges uniformly on $\bar{V}_{m+1}$. This implies that $\left\{f_{n_{k}}\right\}$ is the converging sequence in $\mathcal{O}_{c}^{m+1}\left(V_{m+1}\right)$. Therefore the mapping from $\mathcal{O}_{c}^{m}\left(V_{m}\right)$ to $\mathcal{O}_{c}^{m+1}\left(V_{m+1}\right)$ is compact. This completes the proof.

Next we will show that the space $\mathcal{Q}_{k, l}(K)$ is nuclear. For this purpose, we introduce a new space $\mathcal{O}_{\varepsilon}(\Omega)$ and use the following general theorems. 
Theorem 2. 8. A locally convex space $E$ is nuclear if and only if some, resp. each, fundamental system $U_{\Phi}(E)$ of zero neighbourhood has the following property:

$(P)$ For each zero neighbourhood $U \in U_{ \pm}(E)$ there is a zero neighbourhood $V \in U_{q}(E)$ and a positive Radon measure $\mu$ defined on the weakly compact polar $V^{\circ}$ for which the inequality

$$
P_{U}(x) \leqq \int_{r_{0}}|\langle x, a\rangle| d \mu \quad \text { for all } x \in E
$$

is valid.

Proof. See Proposition 4.1.5 of A. Pietsch [12].

Theorem 2.9. The inductive limit of countably many nuclear locally convex spaces is also nuclear.

Proof. See Proposition 5.2. 4 of A. Pietsch [12].

Definition 2. 10. Let $\Omega$ be an open set in $Q^{k, l}$. We denote by $\mathcal{O}_{\varepsilon}(\Omega)$ the set of all holomorphic functions $f(z)\left(\in \mathcal{O}\left(\Omega \cap C^{n}\right)\right)$ such that $\sup _{z \in K \cap C^{n}}|f(z)| e^{\varepsilon|z|}<\infty$ for any compact set $K$ in $\Omega$. The seminorms of $\mathcal{O}_{\varepsilon}(\Omega)$ is defined by $P_{m}(f)=\sup _{z \in K_{m} \cap \boldsymbol{C}^{n}}|f(z)| e^{\varepsilon(1-1 / m)|z|}$ where $K_{1} \Subset \cdots \Subset K_{m} \Subset \cdots$ is an increasing sequence of compact sets in $\Omega$ which exhaust $\Omega$.

It is clear that the topology is independent of the choice of the sequence of compact sets.

Proposition 2. 11. $O_{\varepsilon}(\Omega)$ is nuclear.

Proof. Let $\rho$ be a positive number such that the polydisc $D=\{z$; $\left.\left|z_{j}-a_{j}\right|<\rho, j=1, \cdots, n\right\}$ is contained in $K_{m+1}$ whenever $a=\left(a_{j}\right) \in K_{m} \cap C^{n}$. Then the following inequality follows from Cauchy's integral formula:

$$
\left(\rho^{2 n} / 2 n\right)|f(a)| \leqq(2 \pi)^{-n} \int_{D}|f(z)| d \lambda,
$$

where $d \lambda$ is the Lebesgue measure on $C^{n}$. 
Then we have for all $a \in K_{m} \cap \boldsymbol{C}^{n}$,

$$
\begin{aligned}
f(a) e^{\varepsilon(1-1 / m)|a|} & \leqq\left(\pi \rho^{2}\right)^{-n} \int_{D}|f(z)| d \lambda e^{\varepsilon(1-1 /(m+1))|a|} \\
& \leqq\left(\pi \rho^{2}\right)^{-n} e^{n \varepsilon \rho} \int_{D}|f(z)| e^{\varepsilon(1-1 /(m+1))|z|} d \lambda \\
& \leqq\left(\pi \rho^{2}\right)^{-n} e^{n \varepsilon \rho} \int_{K_{m+1} \cap C^{n}}|f(z)| e^{\varepsilon(1-1 /(m+1))|z|} d \lambda
\end{aligned}
$$

where we have used the inequality

$$
|a| \leqq|z|+n \rho \quad \text { for } \quad z \in D .
$$

Since the continuous linear forms $\delta_{z}$ with

$$
\left\langle f, \delta_{z}\right\rangle=f(z) e^{\varepsilon(1-1 /(m+1))|z|}
$$

lie in the polar set $V^{\circ}$ of the zero neighbourhood

$$
V=\left\{f \in \mathcal{O}_{\varepsilon}(\Omega) ; P_{m+2}(f) \leqq 1\right\},
$$

it is possible to define a positive Radon measure on $V^{\circ}$ by the equation

$$
\int_{V_{0}} \phi(u) d \mu=\left(\pi \rho^{2}\right)^{-n} e^{n \varepsilon \rho} \int_{K_{m+1} \cap \boldsymbol{C}^{n}} \phi\left(\delta_{z}\right) e^{-\varepsilon|z| /(m+1)(m+2)} d \lambda
$$

for $\phi \in C\left(V^{\circ}\right)$ (continuous function on $V^{\circ}$ ) such that the estimate

$$
P_{m}(f) \leqq \int_{V^{\circ}}|\langle f, u\rangle| d \mu \quad \text { for all } f \in \mathcal{O}_{\varepsilon}(\Omega)
$$

holds. Consequently, the locally convex space $\mathcal{O}_{\varepsilon}(\Omega)$ is nuclear by Theorem 2. 8.

Proposition 2. 12. $\mathcal{Q}_{k, l}(K)$ is nuclear for a compact set $K$ in $Q^{k, l}$

Proof. It is clear that $\mathcal{O}_{c}^{m}\left(V_{m}\right)$ is included in $\mathcal{O}_{1 / m}\left(V_{m}\right)$ and $\mathcal{O}_{1 / m}\left(V_{m}\right)$ is included in $\mathcal{O}_{c}^{m+1}\left(V_{m+1}\right)$ and both inclusion mappings are continuous. This shows that

$$
\stackrel{\lim _{\longrightarrow}}{\mathcal{O}_{1 / m}}\left(V_{m}\right)=\lim _{\longrightarrow} \mathcal{O}_{c}^{m}\left(V_{m}\right) \quad\left(=\mathcal{Q}_{k, l}(K)\right) .
$$

Consequently, the space $\mathcal{O}_{k, l}(K)$ is nuclear by Theorem 2.9.

Now we introduce a new function space $\mathscr{I}(\Omega)$ which will be used 
to give a resolution of the sheaf $\widetilde{\mathcal{O}}_{k, l}$.

Definition 2. 13. Let $\Omega$ be an open set in $Q^{k, l}$. We define the space $\mathscr{I}(\Omega)$ of slowly increasing $C^{\infty}$ functions on $\Omega \cap C^{n}$ to be the set of all $C^{\infty}$ functions on $\boldsymbol{\Omega} \cap C^{n}$ which satisfy the following condition:

For any compact set $K$ in $\Omega$ and any positive $\varepsilon$ and positive integer $m$,

$$
\sup _{z \in K \cap \boldsymbol{C}^{n},|\alpha| \leqq m}\left|D^{\alpha} f(x, y)\right| e^{-\varepsilon|z|}<\infty
$$

holds, where $z=x+i y$.

$\mathscr{I}(\Omega)$ becomes a Fréchet space with the seminorms

$$
P_{m}(f)=\sup _{z \in K_{m} \cap \boldsymbol{C}^{n},|\alpha| \leq m}\left|D^{\alpha} f(x, y)\right| e^{-|z| / m}
$$

where $\left\{K_{m}\right\}$ is the increasing sequence of compact sets in $\Omega$ which exhaust $\Omega$

Definition 2. 14. Let $K$ be a compact set in $\boldsymbol{Q}^{k, l}$. We denote by $\mathscr{F}_{c}(K)$ the closed subspace of $\mathscr{F}\left(\boldsymbol{Q}^{k, l}\right)$ whose elements are those functions which have the supports contained in $K$.

Proposition 2. 15. $\mathscr{E}\left(\boldsymbol{Q}^{k, l}\right)$ is a nuclear space.

Proof. Let $\eta(t)$ be the modification of $|t|$ near $t=0$ so as to become $C^{\infty}$ and convex, and put $\eta(t)=\sum_{i=1}^{2 n} \eta\left(t_{i}\right)$ if $t=\left(t_{1}, \cdots, t_{2 n}\right)$. Then the following equality holds

$$
\begin{aligned}
e^{-\varepsilon_{\eta}(t)} f^{(\alpha)}(t) & =\int_{-\infty}^{t_{1}} \frac{\partial}{\partial t_{1}}\left[e^{-\varepsilon \eta(t)} f^{(\alpha)}(t)\right] d t_{1} \\
& =\int_{-\infty}^{t_{1}} \frac{\partial}{\partial t_{1}}\left[\int_{-\infty}^{t_{2}} \frac{\partial}{\partial t_{2}}\left\{e^{-\varepsilon_{\eta}(t)} f^{(\alpha)}(t)\right\} d t_{2}\right] d t_{1} \\
& =\int_{-\infty}^{t_{1}} d t_{1} \int_{-\infty}^{t_{2}} d t_{2} \frac{\partial^{2}}{\partial t_{1} \partial t_{2}}\left(e^{-\varepsilon_{\eta}(t)} f^{(\alpha)}(t)\right) \\
& =\int_{-\infty}^{t_{1}} d t_{1} \cdots \int_{-\infty}^{t_{2 n}} d t_{2 n} \frac{\partial^{2 n}}{\partial t_{1} \cdots \partial t_{2 n}}\left(e^{-\varepsilon \eta(t)} f^{(\alpha)}(t)\right) \\
& =\int_{-\infty}^{t_{1}} d t_{1} \cdots \int_{-\infty}^{t_{2 n}} d t_{2 n}\left[\prod_{i=1}^{2 n}\left(\frac{\partial}{\partial t_{i}}-\varepsilon \eta^{\prime}\left(t_{i}\right)\right) f^{(\alpha)}(t)\right] e^{-\varepsilon \eta(t)} .
\end{aligned}
$$


Since $|t| \leqq \eta(t) \leqq|t|+\delta$ and $\left|\eta^{\prime}(t)\right| \leqq C^{\prime}$ for some positive $\delta$ and $C^{\prime}$, we have the estimate

$$
\left|e^{-\varepsilon|t|} f^{(\alpha)}(t)\right| \leqq C \int_{-\infty}^{\infty} \cdots \int_{-\infty}^{\infty} e^{-\varepsilon|t|} \sum_{|\beta| \leqq|\alpha|+2 n}\left|f^{(\beta)}(t)\right| d t_{1} \cdots d t_{2_{n}}
$$

for some constant $C$.

Since the continuous linear forms $\varepsilon_{t}^{[\alpha]}$ with $\left\langle f, \varepsilon_{t}^{[\alpha]}\right\rangle=e^{-|t| / m+2 n} f^{(\alpha)}(t)$ for $|\alpha| \leqq m+2 n$ are in the polar set $V^{\circ}$ of the zero neighbourhood $V=\left\{f \in \mathscr{I}\left(\boldsymbol{Q}^{k, l}\right) ; P_{m+2 n}(f) \leqq 1\right\}$, it is possible to define a positive Radon measure on $V^{\circ}$ by

$$
\int_{V^{\circ}} \phi(a) d \mu=C \sum_{|\alpha| \leqq m+2 n} \int_{-\infty}^{\infty} \cdots \int_{-\infty}^{\infty} \phi\left(\varepsilon_{t}^{[\alpha]}\right) e^{-2 n|t| / m(m+2 n)} d t_{1} \cdots d t_{2 n}
$$

for $\phi \in C\left(V^{\circ}\right)$ so that the estimate

$$
P_{m}(f) \leqq \int_{v^{\circ}}|\langle f, a\rangle| d \mu
$$

is valid. Consequently, the locally convex space $\mathscr{I}\left(\boldsymbol{Q}^{k, l}\right)$ is nuclear by Theorem 2.8.

In order to show that $\mathscr{I}(\Omega)$ is nuclear, we use the following theorems.

Theorem 2. 16. Each linear subspace $F$ of a nuclear locally convex space $E$ is also nuclear.

Proof. See Proposition 5.1.1 of A. Pietsch [12].

Theorem 2. 17. The locally convex kernel of arbitrarily many nuclear locally convex spaces is also nuclear.

Proof. See Theorem 5.2.3 of A. Pietsch [12].

Theorem 2. 18. For a compact set $K$ in $Q^{k, l}, \mathscr{I}_{c}(K)$ is a nuclear space.

Proof. Since $\mathscr{F}_{c}(K)$ is a closed subspace of $\mathscr{E}\left(Q^{k, l}\right), \mathscr{I}_{c}(K)$ is 
nuclear by Proposition 2.15 and Theorem 2.16.

Theorem 2. 19. For an open set $\Omega$ in $Q^{k, l}, \mathscr{F}(\Omega)$ is a Fréchet nuclear space.

Proof. Let $\phi_{m}(t)$ be a $C^{\infty}$ function whose support is contained in $K_{m} \cap \boldsymbol{C}^{n}$ where their derivatives are bounded, and $\phi_{m}(t)=1$ in $K_{m-1} \cap \boldsymbol{C}^{n}$. Then the topology of $\mathscr{I}(\Omega)$ is the kernel topology of the mappings $\mathscr{F}(\Omega) \ni f \rightarrow \phi_{m} f \in \mathscr{F}_{c}\left(K_{m}\right)$. Since $\mathscr{F}_{c}\left(K_{m}\right)$ are nuclear, $\mathscr{F}(\Omega)$ is also nuclear by Theorem 2.17. It is known that $\mathscr{I}(\Omega)$ is a Fréchet space.

Definition 2. 20. Let $\Omega$ be an open set in $Q^{k, l}$. If $\phi$ is a continuous function in $\Omega \cap C^{n}$, we denote by $L^{2}(\Omega, \phi)$ the space of functions in $\Omega \cap C^{n}$ which are square integrable with respect to the measure $e^{-\phi} d \lambda$, where $d \lambda$ is the Lebesgue measure in $\boldsymbol{C}^{n}$.

Definition 2. 21. Let $\Omega$ be an open set in $\boldsymbol{Q}^{k, l}$. We denote by $L^{2}(\Omega, \phi$, loc $)$ the space of functions in $\Omega \cap C^{n}$ which are square integrable on $K \cap C^{n}$ for all compact set $K$ in $\Omega$ with respect to the measure $e^{-\phi} d \lambda$.

Definition 2. 22. Let $\Omega$ be an open set in $\boldsymbol{Q}^{k, l}$. We denote by $W^{s}(\Omega, \phi)$ and $W^{s}(\Omega, \phi$, loc $)$, where $s$ is a nonnegative integer, are the sets of functions in $\Omega \cap C^{n}$ whose derivatives of order $\leqq s$ are in $L^{2}(\Omega, \phi)$ and $L^{2}(\Omega, \phi$, loc $)$ respectively.

Definition 2. 23. Let $\Omega$ be an open set in $\boldsymbol{Q}^{k, l}$. We denote by $H^{s}(\Omega, \phi)$ the closure of $C_{0}^{\infty}\left(\Omega \cap C^{n}\right)$ in $W^{s}(\Omega, \phi)$.

Proposition 2. 24. $W^{\prime}(\Omega, \phi$, loc $)$ is an $F S^{*}$ space.

Proof. Let $K_{1} \subset K_{2} \Subset \cdots$ be an increasing sequence of compact sets in $\Omega$ which exhaust $\Omega$. Let $\phi_{m}(z)$ be a $C^{\infty}$ function with bounded derivatives whose support is contained in $\check{K}_{m} \cap \boldsymbol{C}^{n}$ and $\phi_{m}(z)=1$ in $K_{m-1} \cap \boldsymbol{C}^{n}$. For $f \in W^{s}(\Omega, \phi$, loc $), \phi_{m} f$ belongs obviously to $W^{s}\left(\check{K}_{m}, \phi\right)$, but moreover $\phi_{m} f$ belongs to $H^{s}\left(\stackrel{\circ}{K}_{m}, \phi\right)$. In fact, we can choose functions $\alpha_{\nu}(z)$, 
$\nu=1,2, \cdots$, such that $\alpha_{\nu}(z)=1$ if $|z| \leqq \nu$ and $\alpha_{\nu}(z)=0$ if $|z| \geqq \nu+1$ and their derivatives are bounded uniformly in $\nu$. Then $\alpha_{\nu} \phi_{m} f$ converges to $\phi_{m} f$ in $W^{s}\left(\AA_{m}, \phi\right)$ as $\nu$ tends to infinity. Since the support of $\alpha_{\nu} \phi_{m} f$ is compact, by using the Friedrichs mollifier, we can approximate $\alpha_{\nu} \phi_{m} f$ by functions belonging to $C_{0}^{\infty}\left(\stackrel{\circ}{K}_{m} \cap \boldsymbol{C}^{n}\right)$ in $W^{s}\left(\stackrel{\circ}{K}_{m}, \phi\right)$. Thus we have proved that

$$
W^{s}(\Omega, \phi, \operatorname{loc})=\lim H^{s}\left(\stackrel{\circ}{K}_{m}, \phi\right) .
$$

The proof is complete if we show that the mapping $H^{s}\left(K_{m+1}^{\circ}, \phi\right) \ni f$ $\rightarrow \phi_{m} f \in H^{s}\left(\AA_{m}, \phi\right)$ is weakly compact, but it is obvious since the bounded set in the Hilbert space is relatively weakly compact.

Proposition 2. 25. $\mathscr{I}(\Omega)=\lim _{\longleftarrow} W^{s}(\Omega,|z| / m$, loc $)$.

Proof. Let $\mathscr{I}^{m}(\Omega)$ be a Fréchet space which consists of $C^{m}$ functions $f$ on $\Omega \cap C^{n}$ such that $P_{K}^{m}(f)$ are finite for any compact set $K$ in $\Omega$, where $P_{K}^{m}(f)=\sup _{z \in \mathbb{K}_{n} \boldsymbol{C}^{n}|\alpha| \leq m}\left|D^{\alpha} f(x, y)\right| e^{-|z| / m}$ are the seminorms of $\mathscr{F}^{m}(\Omega)$. Then the inclusion $\mathscr{F}^{m+1}(\Omega) \subset W^{m}(\Omega,|z| / m$, loc $)$ is clear and from the well-known Sobolev lemma we have $W^{m+2 n}(\Omega,|z| / m$, loc $)$ $\subset \mathscr{I}^{m}(\Omega)$. Moreover, the both inclusion maps are continuous. Therefore it suffices to show that $\mathscr{I}(\Omega)=\lim _{\mathscr{I}} \mathscr{F}^{m}(\Omega)$, but it is clear from the definition of those spaces.

Proposition 2. 26. $\mathscr{I}(\Omega)$ has an $F S^{*}$ space structure.

Proof. From Proposition 2. 23 and Proposition 2.24, we have the following equalities

$$
\begin{aligned}
\mathscr{F}(\Omega) & =\varlimsup_{m} W^{m}(\Omega,|z| / m, \text { loc }) \\
& =\varliminf_{m} \varliminf_{n} H^{m}\left(\stackrel{\circ}{K}_{n},|z| / m\right) \\
& =\longleftarrow_{m} H^{m}\left(\stackrel{\circ}{K}_{m},|z| / m\right) .
\end{aligned}
$$

Since the mapping from $H^{m+1}\left(\stackrel{\circ}{K}_{m+1},|z| / m+1\right)$ into $H^{m}\left(\AA^{\circ},|z| / m\right)$ is weakly compact $\mathscr{I}(\Omega)$ is an $F S^{*}$ space. 
Remark 2.27. Since $\mathscr{I}(\Omega)$ is a nuclear Fréchet space, $\mathscr{I}(\Omega)$ has an $F S$ space structure.

From Proposition 2.25 we have the following equalities

$$
\begin{aligned}
(\mathscr{I}(\Omega))^{\prime} & ={\lim _{m}}_{\longrightarrow}\left[W^{m}(\Omega,|z| / m, \text { loc })\right]^{\prime} \\
& =\stackrel{\lim }{m}_{m} \varliminf_{\rightarrow}\left[H^{m}\left(\stackrel{\circ}{K}_{n},|z| / m\right)\right]^{\prime} \\
& ={\overleftrightarrow{\lim _{m}}}_{\longrightarrow}\left[H^{m}\left(\dot{\circ}_{m},|z| / m\right)\right]^{\prime} .
\end{aligned}
$$

The following theorem shows that $(\mathscr{I}(\Omega))^{\prime}$ has a $D F S^{*}$ space structure.

Theorem 2. 28. Let $\left\{X_{j}, u_{j k}\right\}$ be a reakly compact projective sequence of Banach spaces such that $u_{j}\left(\lim X_{j}\right)$ is dence in $X_{j}$ for each $j$. Then the dual sequence $\left\{X_{j}^{\prime}, u_{j k}^{\prime}\right\}$ is a weakly compact injective sequence and the strong dual space of the projective limit $\stackrel{\lim }{\longleftarrow} X_{j}$ is isomorphic to the injective limit $\lim _{\longrightarrow} X_{j}^{\prime}$.

Proof. See Theorem 11 of H. Komatsu [7].

Proposition 2.29. For each bounded set $B$ in $(\mathscr{F}(\Omega))^{\prime}$, there is an index $m$ such that $B$ is the image $u_{m}\left(B_{m}\right)$ of bounded set $B_{m}$ in $\left[H^{m}\left(\stackrel{\circ}{K}_{m},|z| / m\right)\right]^{\prime}$, where $u_{m}$ is an inclusion mapping from $\left[H^{m}\left(\stackrel{\circ}{K}_{m},|z| / m\right)\right]^{\prime}$ into $(\mathscr{F}(\Omega))^{\prime}$, and $u_{m}$ is a reak homeomorphism of $B_{m}$ onto $B$.

Proof. This proposition is a direct consequence of the following theorem.

Theorem 2. 30. The injective limit $X=\underline{\lim } X_{j}$ of a weakly compact sequence of locally convex spaces is a complete reflexive and bornologic $D F$ space. For each bounded set $B$ in $X$ there is an index $k$ such that $B$ is the image $u_{k}\left(B_{k}\right)$ of a bounded set $B_{k}$ in $X_{k}$ and $u_{k}$ is a weak homeomorphism of $B_{k}$ onto $B$. In particular, a sequence $x_{n}$ in $X$ converges weakly to zero if and only if there is a 
sequence $y_{n}$ in $X_{k}$ with $x_{n}=u_{k}\left(y_{n}\right)$ which converges weakly to zero in $X_{k}$.

Proof. See Theorem 6 of H. Komatsu [7].

Proposition 2. 31. Let $\left[H^{m}(\Omega, \phi)\right]^{\prime}$ be the dual space of $H^{m}(\Omega, \phi)$. Then $T \in\left[H^{m}(\Omega, \phi)\right]^{\prime}$ has the form

$$
T=\sum_{|\alpha| S m} D^{\alpha} f_{\alpha}(x), \quad f_{\alpha}(x) \in L^{2}(\Omega,-\phi) .
$$

Conversely the distribution having the above form belongs to $\left[H^{m}(\Omega, \phi)\right]^{\prime}$.

Proof. For $T \in\left[H^{m}(\Omega, \phi)\right]^{\prime}$ there exists $g \in H^{m}(\Omega, \phi)$ from the Riesz theorem such that the following equation holds

$$
\langle T, f\rangle=(g, f)_{H^{m}(\Omega, \phi)}=\sum_{|\alpha| \leqq m}\left(D^{\alpha} g, D^{\alpha} f\right)_{L^{2}(\Omega, \phi)} .
$$

In particular if $f \in C_{0}^{\infty}\left(\Omega \cap C^{n}\right)$ we have

$$
\langle T, f\rangle=\sum_{|\alpha| \leqq m}(-1)^{|\alpha|}\left\langle D^{\alpha}\left(e^{-\phi(x)} D^{\alpha} \overline{g(x)}\right), f(x)\right\rangle .
$$

Since $C_{0}^{\infty}\left(\Omega \cap \boldsymbol{C}^{n}\right)$ is dense in $H^{m}(\Omega, \phi)$ and $g \in H^{m}(\Omega, \phi)$, we have

$$
T=\sum_{|\alpha| \leqq m} D^{\alpha}\left((-1)^{|\alpha|} e^{-\phi(x)} D^{\alpha} \overline{g(x)}\right)
$$

and

$$
(-1)^{|\alpha|} e^{-\phi(x)} D^{\alpha} \overline{g(x)}=f_{\alpha}(x) \in L^{2}(\Omega,-\phi) .
$$

Thus we have the required form (2.1) of $T$. Conversely, for the distribution having the form (2.1) we have

$$
\langle T, f\rangle=\sum_{|\alpha| \leqq m}\left\langle D^{\alpha} f_{\alpha}(x), f(x)\right\rangle=\sum_{|\alpha| \leqq m}(-1)^{|\alpha|}\left\langle f_{\alpha}(x), D^{\alpha} f(x)\right\rangle
$$

for $f \in C_{0}^{\infty}\left(\Omega \cap C^{n}\right)$. Since $C_{0}^{\infty}\left(\Omega \cap C^{n}\right)$ is dense in $H^{m}(\Omega, \phi)$ we have for $f \in H^{m}(\Omega, \phi)$

$$
\langle T, f\rangle=\sum_{|\alpha| \Sigma_{m}}(-1)^{|\alpha|}\left\langle f_{\alpha}(x), D^{\alpha} f(x)\right\rangle .
$$

It is obvious that the above linear form on $H^{m}(\Omega, \phi)$ is continuous. This completes the proof. 
Remark 2.32. The topology of $\left[H^{m}(\Omega, \phi)\right]^{\prime}$ is given by the norm,

$$
\|T\|_{m, L 2(\Omega,-\phi)}^{2}=\sum_{|\alpha| \leqq m}\left\|f_{\alpha}(x)\right\|_{L^{2}(\Omega,-\phi)}^{2}
$$

for $T$ of the form (2.1).

\section{§ 3. Approximation Theorem}

Theorem 3. 1. Let $K$ be a compact set in $\prod_{i=1}^{j} \boldsymbol{D}^{n_{i}}$, then $\mathcal{Q}_{k, l}\left(\prod_{i=1}^{j} \boldsymbol{D}^{n_{i}}\right)$ is dense in $\mathcal{O}_{k, l}(K)$.

Proof. We define $U_{p}=\prod_{i=1}^{j} U_{p}^{k_{i}, l_{i}}$ where

$$
U_{p}^{k_{i}, l_{i}}=\left\{z_{i}^{\prime} \in C^{n_{i}} ;\left|\operatorname{Im} \underline{z}_{k_{i}}\right|^{2}<\left(\left|\operatorname{Re} z_{i}^{\prime}\right|^{2}+1\right) / p,\left|\operatorname{Im} \underline{z}_{l_{i}}\right|^{2}<1 / p\right\}
$$

with $n_{i}=k_{i}+l_{i}$ and $z_{i}^{\prime}=\left(\underline{z}_{k_{i}}, \underline{z}_{l_{i}}\right) \in \boldsymbol{Q}^{k_{i}, l_{i}}$. The proof goes on in a fashion similar to that in Theorem 2.2.1 of T. Kawai [6], if we can construct a sequence of subsets $\left\{\Omega_{p}\right\}$ possessing the following properties:

(a) $U_{p} \supset \Omega_{p} \supseteq K$ and $\Omega_{p}$ 's tend decreasingly to $K$.

(b) For any $p$ and any $T\left(\subset \Omega_{p}\right)$, there exists an open set $V$ and a function $\theta(z)$ strictly plurisubharmonic in $U_{p}$ such that

(i) $T \subset V \Subset \Omega_{p}$;

(ii) $\theta(z)<0$ on $T \cap \boldsymbol{C}^{n}$;

(iii) $\theta(z)>0$ near $\partial V \cap C^{n}$;

(iv) $\sup _{L \cap \boldsymbol{C}^{n}} \theta(z) \leqq M_{L}<\infty$ for any $L \subset \Omega_{p}$.

We can also construct $\left\{\Omega_{p}\right\}$ in a way similar to that in Theorem 2.2.1 of T. Kawai [6] or Appendix of S. Nagamachi and N. Mugibayashi [11]. We shall say that $\Omega$ is of type (E) if $\Omega=\cap_{l=1}^{\infty} V^{\imath}$, where $V^{\imath}=\stackrel{\circ}{U}^{l}$ and

$$
\begin{gathered}
U^{l}=\left\{z \in C^{n} ;\left|f_{l}(z)\right|<1, d_{l}\left|\operatorname{Im} \underline{z}_{k_{i}}\right|^{2}-\left|\operatorname{Re} z_{i}^{\prime}\right|^{2}<1\right. \\
\left.d_{l}\left|\operatorname{Im} \underline{z}_{l_{i}}\right|^{2}<1,1 \leqq i \leqq j\right\}
\end{gathered}
$$

Here $f_{l}(z)=c_{l} \exp \left\{-\Sigma_{j}\left(z_{j}-a_{j}^{(l)}\right)^{2}\right\}, c_{l} \in \boldsymbol{R}$ and $a_{j}^{(l)} \in \boldsymbol{R}$. Then $K$ can be approximated by a decreasing sequence of $\Omega_{p}$ of type (E). Moreover, for any $T \subset \Omega_{p}$ we can find an open set $V$ of type (E) such that $T \subset V \in \Omega_{p}$. In the construction of $V$, we may assume $d_{l}=d$. On setting 


$$
\phi(z)=\max _{i}\left\{d\left|\operatorname{Im} \underline{z}_{k_{i}}\right|^{2}-\left|\operatorname{Re} z_{i}^{\prime}\right|^{2}-1\right\}, \sigma(z)=\sup _{l} \log \left|f_{l}(z)\right|
$$

and

$$
\chi(z)=\max \{\phi(z), \sigma(z)\}
$$

we define

$$
\theta(z)=\chi(z) * \rho_{\varepsilon}+\varepsilon\left[1+\sum_{i}\left\{\log \left(1+\left|\underline{z}_{k_{i}}\right|^{2}\right)+\left|\operatorname{Im} \underline{z}_{l_{i}}\right|^{2}\right\}\right]
$$

where $\rho_{\varepsilon}$ is a mollifier in $\boldsymbol{R}^{2 n}$. Then $\theta(z)$ thus defined is strictly plurisubharmonic and $\theta(z)<0$ on $T \cap \boldsymbol{C}^{n}$ and $\theta(z)>0$ near $\partial V \cap \boldsymbol{C}^{n}$ for sufficiently small $\varepsilon>0$. Thus we have constructed $\left\{\Omega_{p}\right\}$ possessing the properties (a) and (b).

Lemma 3. 2. Let $\mathscr{S}_{1 / 4}^{3 / 4}$ be one of the spaces $\mathscr{S}_{\alpha}^{\beta}$ of type $S$ of I. M. Gelfand and G. E. Shilov [1] having the indices $\alpha=\left(\alpha_{1}, \cdots, \alpha_{n}\right)$, $\beta=\left(\beta_{1}, \cdots, \beta_{n}\right), \alpha_{j}=1 / 4, \beta_{j}=3 / 4$ for $1 \leqq j \leqq n$, and put $\mathscr{Q}_{k, l} \equiv_{\mathcal{Q}_{k, l}}\left(\prod_{i=1}^{j} \boldsymbol{D}^{n_{i}}\right)$, then $\mathscr{S}_{1 / 4}^{3 / 4} \subset \mathscr{P}_{k, l}$ and the original topology of $\mathscr{S}_{1 / 4}^{3 / 4}$ is stronger than that induced by $\mathscr{P}_{k, l}$.

Proof. Let $\mathscr{I}^{m}$ be a Banach space of those entire functions which satisfy the condition

$$
|f|_{m} \equiv \sup _{\boldsymbol{z} \in \boldsymbol{C}^{n}}|f(\boldsymbol{z})| \exp \left\{|\operatorname{Re} z|^{4} / m-m|\operatorname{Im} \boldsymbol{z}|^{4}\right\}<\infty .
$$

$|f|_{m}$ is the norm of the Banach space $\mathscr{I}^{m}$, and $\mathscr{S}_{1 / 4}^{3 / 4}$ is the inductive limit of $\left\{I^{m}\right\}$ (see I. M. Gelfand and G. E. Shilov [1] p. 220). Let

$$
\begin{gathered}
U_{k, l ; m}=\left\{z \in C^{n} ;\left|\operatorname{Im} \underline{z}_{k_{i}}\right|^{2}<\left(1+\left|\operatorname{Re} z_{i}^{\prime}\right|^{2}\right) / m,\right. \\
\left.\left|\operatorname{Im} \underline{z}_{l_{i}}\right|^{2}<1 / m, 1 \leqq i \leqq j\right\}
\end{gathered}
$$

for $z_{i}^{\prime}=\left(\underline{z}_{k_{i}}, \underline{z}_{l_{i}}\right) \in \boldsymbol{Q}^{k_{i}, l_{i}}$ and $\boldsymbol{Q}^{k, l}=\prod_{i=1}^{j} \boldsymbol{Q}^{k_{i}, l_{i}}$, then $\mathcal{P}_{k, l}$ is the inductive limit of $\mathcal{O}_{c}^{m}\left(U_{k, l ; m}\right)$.

If $z \in U_{k, l ; m}(m \geqq 2)$, we have a series of inequalities

$$
\begin{aligned}
|\operatorname{Re} z|^{4} / m-m|\operatorname{Im} z|^{4} & \geqq\left(\frac{1}{m}-\frac{1}{m^{2}}\right)|\operatorname{Re} z|^{4}-\frac{2}{m}\left(j+|\operatorname{Re} z|^{2}\right) \\
& \geqq(|\operatorname{Re} z|+|\operatorname{Im} z|) / m-c \geqq|z| / m-c
\end{aligned}
$$

for some constant $c$. Hence $\|f\|_{m} \leqq e^{c}|f|_{m}$. Therefore $\mathcal{I}^{m} \subset \mathcal{O}_{c}^{m}\left(U_{k, l ; m}\right)$ 
and consequently $\mathscr{S}_{1 / 4}^{3 / 4} \subset \mathscr{Q}_{k, l}$ and the topology of $\mathscr{S}_{1 / 4}^{3 / 4}$ induced by $\mathscr{Q}_{k, l}$ is weaker than its original topology.

Proposition 3. 3. $\mathscr{S}_{1 / 4}^{3 / 4}$ is dense in $\mathscr{Q}_{k, l}$.

Proof. See Proposition 2.2 of S. Nagamachi and N. Mugibayashi [10].

Corollary 3.4. Let $n=\sum_{i=1}^{j} k_{i}+l_{i}, \mathscr{P}_{*}=\mathscr{Q}_{0, n}$ and $\mathscr{P}_{* *}=\mathscr{P}_{n, 0 .}$ Then $\mathscr{Q}_{* *}$ is densely included in $\mathscr{P}_{k, l}$ and $\mathscr{Q}_{k, l}$ is densely included in $\mathscr{Q}_{*}$.

Proof. Since the inclusion relation is obvious from the definition, this corollary is the direct consequence of Proposition 3. 3.

Proposition 3. 5. $\bigotimes_{i=1}^{j} \mathcal{P}_{k_{i}, l_{i}}$ is dense in $\mathscr{P}_{k, l}$.

Proof. B. S. Mityagin [8] has shown that $\bigotimes_{i=1}^{j} \mathscr{S}_{1 / 4}^{3 / 4}\left(\boldsymbol{R}^{n_{i}}\right)$ is dense in $\mathscr{S}_{1 / 4}^{3 / 4}\left(\boldsymbol{R}^{n}\right) \quad\left(n=\sum n_{i}\right) . \quad$ By Lemma 3.2, $\bigotimes_{i=1}^{j} \mathscr{S}_{1 / 4}^{3 / 4}\left(\boldsymbol{R}^{n_{i}}\right) \subset \bigotimes_{i=1}^{j} \mathscr{Q}_{k_{i}, l_{i}}$ and the topology of $\mathscr{S}_{1 / 4}^{3 / 4}\left(\boldsymbol{R}^{n}\right)$ induced by $\mathscr{Q}_{k, l}$ is weaker than its original topology, and $\mathscr{S}_{1 / 4}^{3 / 4}\left(\boldsymbol{R}^{n}\right)$ is dense in $\mathscr{Q}_{k, l}$ by Proposition 3. 3. Therefore $\bigotimes_{i=1}^{j} \mathscr{P}_{k_{i}, l_{i}}$ is dense in $\mathscr{Q}_{k, l}$.

Proposition 3. 6. $\hat{\bigotimes}_{i=1}^{j} \mathcal{Q}_{k_{i}, l_{i}}=\mathscr{P}_{k, l}$.

Proof. By Proposition 3.5 and Proposition 2.12, it suffices to show that $\mathscr{Q}_{k, l}$ induces on $\bigotimes_{i=1}^{j} \mathscr{Q}_{k_{i}, l_{i}}$ the topology $\pi=\varepsilon$. Since the multilinear mapping $\left(u_{1}, \cdots, u_{j}\right) \rightarrow \bigotimes_{i=1}^{j} u_{i}$ of $\prod_{i=1}^{j} \mathscr{P}_{k_{i}, l_{i}}$ into $\mathscr{P}_{k, l}$ is continuous, the $\pi$ topology is finer than the induced topology. On the other hand, if $\left\{f_{\nu}\right\}$ is a net converging to zero in $\mathscr{Q}_{k, l}$ it converges to zero uniformly on the equicontinuous subset of $\left(\mathscr{Q}_{k, l}\right)^{\prime}$. In particular, sets of the form $\bigotimes_{i=1}^{j} A_{i}^{\prime}$, with equicontinuous sets $A_{i}^{\prime} \subset\left(\mathscr{P}_{k_{i}, l_{i}}\right)^{\prime}$ are equicontinuous in $\left(\mathscr{P}_{k, l}\right)^{\prime}$. Thus $\mathcal{Q}_{k, l}$ induces on $\bigotimes_{i=1}^{j} \mathscr{Q}_{k_{i}, l_{i}}$ a topology which is finer than the $\varepsilon$ one. 
Remark 3. 7. Let $\boldsymbol{D}^{n}$ and $\prod_{i=1}^{j} \boldsymbol{D}^{n_{i}}$ be closures of $\boldsymbol{R}^{n}$ in $\boldsymbol{Q}^{0 . n}$ and $\prod_{i=1}^{j} \boldsymbol{Q}^{0, n_{i}}$, where $n=\sum_{i=1}^{j} n_{i}$. Then $\mathcal{Q}_{0, n}\left(\boldsymbol{D}^{n}\right)=\mathcal{Q}_{k, l}\left(\prod_{i=1}^{j} \boldsymbol{D}^{n_{i}}\right)$, where $\left(k_{i}, l_{i}\right)$ $=\left(0, n_{i}\right)$. In fact the fundamental system of neighbourhoods of $\boldsymbol{R}^{n}$ is given by $U_{m}=\left\{z \in C^{n} ;|\operatorname{Im} z|<1 / m\right\}$ in both topologies.

\section{§4. The Soft Resolutions of the Sheaf $\widetilde{\mathcal{O}}_{k, l}$}

In this section we construct a soft resolution of the sheaf $\widetilde{\mathcal{O}}_{k, l}$ by slowly increasing $C^{\infty}$ differential forms, which will be used later to calculate the cohomology groups $H^{s}\left(V, \widetilde{\mathcal{O}}_{k, l}\right)$.

Let $\Omega$ be an open set in $C^{n}$. If $\phi$ is a continuous function in $\Omega$, we denote by $L_{(p, q)}^{2}(\Omega, \phi)$ the space of forms of type $(p, q)$ with coefficients in $L^{2}(\Omega, \phi)$,

$$
f=\sum_{|T|=p}^{\prime} \sum_{|J|=q}^{\prime} f_{I, J} d z^{I} \wedge d \bar{z}^{J}
$$

where $\Sigma^{\prime}$ means that the summation is performed only over strictly increasing multiindices. We set

$$
|f|^{2}=\sum_{I, J}^{\prime}\left|f_{I, J}\right|^{2}
$$

and

$$
\|f\|_{\phi}^{2}=\int_{\Omega_{\cap} \boldsymbol{C}^{n}}|f|^{2} e^{-\phi} d \lambda .
$$

It is clear that $L^{2}(\Omega, \phi)$ is a Hilbert space with this norm. Similarly we define $D_{(p, q)}(\Omega)$ where $D(\Omega)$ is a notation for $C_{0}^{\infty}(\Omega)$.

If $\phi_{1}$ and $\phi_{2}$ are two continuous functions in $\Omega$, then the CauchyRiemann operator $\bar{\partial}$ defines a linear, closed, densely defined operator

$$
T: L_{(p, q)}^{2}\left(\Omega, \phi_{1}\right) \rightarrow L_{(p, q+1)}^{2}\left(\Omega, \phi_{2}\right)
$$

whose domain contains $D_{(p, q)}(\Omega)$. Let $\phi_{3}$ be another continuous function and let $S$ be the operator from $L_{(p, q+1)}^{2}\left(\Omega, \phi_{2}\right)$ to $L_{(p, q+2)}^{2}\left(\Omega, \phi_{3}\right)$ defined by $\bar{\partial}$.

Lemma 4. 1 (Hörmander). Let $\eta_{\nu}, \nu=1,2, \cdots$, be a sequence of functions in $C_{0}^{\infty}(\Omega)$ such that $0 \leqq \eta_{\nu} \leqq 1$ and $\eta_{\nu}=1$ on any compact subset of $\Omega$ when $\nu$ is large. Suppose that $\phi_{2} \in C^{1}(\Omega)$ and that 


$$
e^{-\phi_{j+1}} \sum_{k=1}^{n}\left|\partial \eta_{\nu} / \partial \bar{z}_{k}\right| \leqq e^{-\phi_{j}} \quad j=1,2 ; \nu=1,2, \cdots .
$$

Then $D_{(p, q+1)}(\Omega)$ is dense in $D_{T^{*}} \cap D_{S}$ for the graph norm

$$
f \rightarrow\|f\|_{\phi_{2}}+\left\|T^{*} f\right\|_{\phi_{1}}+\|S f\|_{\phi_{s}} .
$$

Proof. See Lemma 4.1.3 of L. Hörmander [2].

Lemma 4.2. Let $\phi$ be a $C^{2}$-plurisubharmonic function on $\boldsymbol{C}^{n}$ such that $\partial \phi / \partial z_{j}, \partial \phi / \partial \bar{z}_{j}$ and $\partial^{2} \phi / \partial z_{j} \partial \bar{z}_{j}(0 \leqq j \leqq n)$ are bounded. If $S f \in L_{(p, q+2)}^{2}\left(\boldsymbol{C}^{n}, \phi\right)$ and $T^{*} f \in L_{(p, q)}^{2}\left(\boldsymbol{C}^{n}, \phi\right)$ for $f \in L_{(p, q+1)}^{2}\left(\boldsymbol{C}^{n}, \phi\right)$, then $f \in W_{(p, q+1)}^{1}\left(\boldsymbol{C}^{n}, \phi\right)$.

Proof. Choose a function $\psi \in C^{\infty}\left(\boldsymbol{C}^{n}\right)$ such that

$$
\sum_{k=1}^{n}\left|\partial \eta_{\nu} / \partial \bar{z}_{k}\right| \leqq e^{\psi} \quad \text { in } \quad C^{n}, \nu=1,2, \cdots .
$$

If we set $\phi_{1}=\phi-2 \psi, \phi_{2}=\phi-\psi, \phi_{3}=\phi$, the condition (4.1) is satisfied for any choice of $\phi$, and the following inequality holds for $f \in D_{(p, q+1)}\left(\boldsymbol{C}^{n}\right)$ :

$$
\begin{gathered}
\sum_{I, K}^{\prime} \int \sum_{j, k=1}^{n} f_{I, j K} \overline{f_{I, k K}} \partial^{2} \phi / \partial z_{j} \partial \bar{z}_{k} e^{-\phi} d \lambda+\sum_{T, J}^{\prime} \sum_{j=1}^{n} \int\left|\partial f_{I, J} / \partial \bar{z}_{j}\right|^{2} e^{-\phi} d \lambda \\
\leqq 2\left\|T^{*} f\right\|_{\phi_{1}}^{2}+\|S f\|_{\phi_{\mathrm{s}}}^{2}+2 \int|f|^{2}|\partial \phi|^{2} e^{-\phi} d \lambda
\end{gathered}
$$

((4. 2.7) in L. Hörmander [2]). Since we can choose $\eta_{y}$ satisfying the condition

$$
\sum_{k=1}^{n}\left|\partial \eta_{\nu} / \partial \bar{z}_{k}\right| \leqq 1, \quad \nu=1,2, \cdots,
$$

we can choose $\psi=0$ in (4.2). Since $\phi$ is a $C^{2}$-plurisubharmonic function, we have for $f \in D_{(p, q+1)}\left(C^{n}\right)$

$$
\sum_{I, J}^{\prime} \sum_{j=1}^{n} \int\left|\partial f_{I, J} / \partial \bar{z}_{j}\right|^{2} e^{-\phi} d \lambda \leqq 2\left\|T^{*} f\right\|_{\phi}^{2}+\|S f\|_{\phi}^{2} .
$$

If $f$ only satisfies the hypothesis in the lemma, we can choose $f_{n} \in D_{(p, q+1)}\left(\boldsymbol{C}^{n}\right)$ such that $f_{n} \rightarrow f, T^{*} f_{n} \rightarrow T^{*} f$ and $S f_{n} \rightarrow S f$ as $n \rightarrow \infty$ by Lemma 4. 1. Then $\partial f_{n, I, J} / \partial \bar{z}_{j}$ converges in $L^{2}\left(\boldsymbol{C}^{n}, \phi\right)$ and hence $\partial f_{I, J} / \partial \bar{z}_{j}$ $\in L^{2}\left(\boldsymbol{C}^{n}, \phi\right)$. The proof is therefore reduced to the following lemma. 
Lemma 4. 3. Let $\phi$ be a $C^{2}$-function on $C^{n}$ such that $\partial \phi / \partial z_{j}$, $\partial \phi / \partial \bar{z}_{j}$ and $\partial^{2} \phi / \partial z_{j} \partial \bar{z}_{j}(0 \leqq j \leqq n)$ are bounded. If $\partial w / \partial \bar{z}_{k} \in L^{2}\left(\boldsymbol{C}^{n}, \phi\right)$ $k=1,2, \cdots, n$ for $w \in L^{2}\left(\boldsymbol{C}^{n}, \phi\right)$, then $w \in W^{1}\left(\boldsymbol{C}^{n}, \phi\right)$.

Proof. We have only to prove that $\partial w / \partial z_{j} \in L^{2}\left(C^{n}, \phi\right)$. If $w \in C_{0}^{\infty}\left(\boldsymbol{C}^{n}\right)$, integrations by parts give the following equality,

$$
\begin{aligned}
& \int\left|\partial w / \partial z_{j}\right|^{2} e^{-\phi(z)} d \lambda \\
& =\int\left\{\left|\partial w / \partial \bar{z}_{j}\right|^{2}-\partial w / \partial \bar{z}_{j} \partial \phi / \partial z_{j} \bar{w}-w \partial \phi / \partial \bar{z}_{j} \overline{\partial w / \partial \bar{z}_{j}}\right. \\
& \left.\quad+\left(\partial \phi / \partial \bar{z}_{j} \partial \phi / \partial z_{j}-\partial^{2} \phi / \partial z_{j} \partial \bar{z}_{j}\right)|w|^{2}\right\} e^{-\phi(z)} d \lambda .
\end{aligned}
$$

From the assumption, $\partial \phi / \partial z_{j}, \partial \phi / \partial \bar{z}_{j}$ and $\partial^{2} \phi / \partial z_{j} \partial \bar{z}_{j}$ are bounded. Therefore we have

$$
\left\|\partial w / \partial z_{j}\right\|_{\phi}^{2} \leqq C\left(\left\|\partial w / \partial \bar{z}_{j}\right\|_{\phi}+\|w\|_{\phi}\right)^{2}
$$

for some positive constant $C$. If $w$ only satisfies the hypotheses in Lemma 4. 3, approximating $w$ by $w_{n} \in D\left(\boldsymbol{C}^{n}\right)$ in the same way as we did in the proof of the previous lemma, we obtain that $\partial w / \partial z_{j} \in L^{2}\left(\boldsymbol{C}^{n}, \phi\right)$.

Let $\Omega$ be a pseudoconvex domain in $\boldsymbol{C}^{n}$ and $\psi(z)$ be a plurisubharmonic function in $\Omega$. We denote by $X_{j}, Y_{j}$ and $Z_{j}$ the spaces of differential forms $L_{(p, q-1)}^{2}\left(\Omega, \phi_{1}\right), L_{(p, q)}^{2}\left(\Omega, \phi_{2}\right), L_{(p, q+1)}^{2}\left(\Omega, \phi_{3}\right)$ respectively, where

$$
\begin{aligned}
& \phi_{1}(z)=\eta(z) / j+4 \log \left(1+|z|^{2}\right)+\psi(z), \\
& \phi_{2}(z)=\eta(z) / j+2 \log \left(1+|z|^{2}\right)+\psi(z)
\end{aligned}
$$

and

$$
\phi_{8}(z)=\eta(z) / j+\psi(z) .
$$

Here, we denote by $\eta(z)$ the modification of $|z|$ near $\left\{z_{j}=0\right.$ for some $\left.j\right\}$ so as to become $C^{\infty}$ and convex, which has been used in the proof of Proposition 2. 15.

Lemma 4. 4. Let $T$ and $S$ be the Cauchy-Riemann operator defined in the distribution sense, then the sequence

$$
X_{j} \stackrel{T}{\longrightarrow} Y_{j} \stackrel{S}{\longrightarrow} Z_{j} \text { is exact. }
$$


Proof. Since $\eta(z) / j+2 \log \left(1+|z|^{2}\right)+\psi$ is a plurisubharmonic function, this lemma follows from Theorem 4.4.2 of L. Hörmander [2].

Definition 4. 5. An open set $\Omega$ in $\boldsymbol{Q}^{k, l}$ is said to be a $Q^{k, l}$-pseudoconvex domain if it satisfies the conditions:

$$
\sup _{z \in \Omega \cap \boldsymbol{C}^{n}, 1 \leqq i \leqq j}\left\{2\left|\operatorname{Im} \underline{z}_{k_{i}}\right|^{2}-\left|\operatorname{Re} z_{i}^{\prime}\right|^{2},\left|\operatorname{Im} \underline{z}_{l_{i}}\right|^{2}\right\} \leqq M<\infty,
$$

where $z_{i}^{\prime}=\left(\underline{z}_{k_{i}}, \underline{z}_{l_{i}}\right) \in Q^{k_{i}, l_{i}}$ and $\boldsymbol{Q}^{k, l}=\prod_{i=1}^{j} \boldsymbol{Q}^{k_{i}, l_{i}}$.

(ii) There exists a $C^{\infty}$ strictly plurisubharmonic function $\theta(z)$ on $\Omega \cap C^{n}$ having the properties: any $L \in \mathcal{E}$,

(a) $\{z ; \theta(z)<c\} \Subset \Omega$ for any $c<\infty$ and $\sup _{L \cap \boldsymbol{C}^{n}}|\theta(z)| \leqq M_{L}<\infty$ for

(b) $\sup _{L \cap \boldsymbol{C}^{n}}\left|D^{\alpha} \theta(z)\right| \leqq M_{L, \alpha}$ for any multiindex $\alpha$ and $L \subseteq \Omega$.

The $Q^{k, l}$-pseudoconvex domain is the $\widetilde{\mathscr{O}}_{k, l}$-pseudoconvex domain (Definition 5.1) satisfying the condition (b). In order to get a $C^{\infty}$ resolution of $\widetilde{\mathcal{O}}_{k, l}$, we require the condition.

Let $\Omega$ be a $Q^{k, l}$-pseudoconvex domain. We denote by $A_{j}^{s}, B_{j}^{s}$ and $C_{j}^{s}$, the spaces of differential forms $W_{(p, q-1)}^{s}\left(\Omega, \psi_{1}\right.$, loc $), W_{(p, q)}^{s}\left(\Omega, \psi_{2}\right.$, loc $)$ and $W_{(p, q+1)}^{s}\left(\Omega, \psi_{3}\right.$, loc $)$ respectively, where

$$
\begin{aligned}
& \psi_{1}(z)=\eta(z) / j+4 \log \left(1+|z|^{2}\right), \\
& \psi_{2}(z)=\eta(z) / j+2 \log \left(1+|z|^{2}\right)
\end{aligned}
$$

and

$$
\psi_{3}(z)=\eta(z) / j
$$

Proposition 4. 6. Let $\bar{\partial}$ be the Cauchy-Riemann operator. Then the sequence $A_{j}^{s+1} \stackrel{\bar{\partial}}{\longrightarrow} B_{j}^{s} \stackrel{\bar{\partial}}{\longrightarrow} C_{j}^{s-1}$ is exact.

Proof. (a) First assume that $q=1$. For every $f \in B_{j}^{s}$, we can find a plurisubharmonic function $\psi(z)$ such that $f \in L_{(p, 1)}^{2}\left(\Omega, \phi_{1}\right)$ where $\phi_{1}(z)=\psi_{1}(z)+\psi(z)$, by the assumption of the existence of plurisubharmonic function $\theta(z)$ in Definition 4.5. Therefore the equation $\bar{\partial} u=f$ has a solution $u=\sum^{\prime} u_{I} d z^{I} \in L_{(p, 0)}^{2}\left(\Omega, \psi_{1}\right.$, loc $)$ for every $f \in B_{j}^{s}$ such that $\bar{\partial} f=0$ by Lemma 4. 4. The equation $\bar{\partial} u=f$ means that $\partial u_{I} / \partial \bar{z}_{i}=f_{I, i} \in W^{s}\left(\Omega, \psi_{2}\right.$, loc $)$ 
for all $I$ and $i$. Suppose that $u \in A_{j}^{\sigma}$ for a certain $\sigma$ with $0 \leqq \sigma \leqq s$; we know that this is true if $\sigma=0$. Let $\chi$ be a bounded $C^{\infty}$ function whose all derivatives are bounded and whose support is contained in $K \cap \boldsymbol{C}^{n}$ for some compact set $K$ in $\Omega$. Then we have $\partial \chi u_{I} / \partial \bar{z}_{j}=\chi f_{I, j}+u_{I} \partial \chi / \partial \bar{z}_{j}$ $\in W^{\sigma}\left(\boldsymbol{C}^{n}, \psi_{1}\right)$. If $v$ is a derivative of order $\sigma$ of $\chi u_{I}$, then $\partial v / \partial \bar{z}_{j}$ $\in L^{2}\left(\boldsymbol{C}^{n}, \psi_{1}\right)$ for every $j$. Hence $v \in W^{1}\left(\boldsymbol{C}^{n}, \psi_{1}\right)$ by Lemma 4 . 3, that is all derivatives of $\chi u_{I}$ of order $\sigma+1$ are in $L^{2}\left(\boldsymbol{C}^{n}, \psi_{1}\right)$. This means that $u_{I} \in W^{\sigma+1}\left(\Omega, \psi_{1}\right.$, loc $)=A_{j}^{\sigma}$. Repeating the argument, we conclude that $\iota_{I} \in A_{j}^{s+1}$.

(b) Next assume that $q>1$, and define for $(p, q)$ form $f$ $\vartheta f=\sum_{I, K}^{\prime} \sum_{j=1}^{n} \partial f_{I, j K} / \partial z_{j} d z^{I} \bigwedge d \bar{z}^{K}$. Then we have $T^{*} f=(-1)^{p-1} e^{\phi_{1}} \vartheta\left(e^{-\phi_{2}} f\right)$, where $\phi_{j}(z)=\psi_{j}(z)+\psi(z)(j=1,2)$ for the plurisubharmonic function $\psi(z)$ used in the part (a), and $T^{*}$ is the adjoint operator of $T$ in Lemma 4. 4. The solution of the equation $T u=f$ given by Lemma 4.4 can be chosen orthogonal to the null space of $T$, that is, in (the closure of) the range of $T^{*}$. Since $\vartheta^{2}=0$, we have $\vartheta\left(e^{-\phi_{1}} u\right)=0$ or $\vartheta u=a u$ where $a$ is a differential operator of order 0 with $C^{\infty}$ coefficients. Moreover it follows from the property of $\theta(z)$ that the coefficients $a_{I, j}$ of $a$ satisfy the following condition:

$$
\sup _{z \in L \cap C^{n}}\left|D^{\alpha} a_{I, j}(z)\right| e^{\phi_{1}(z)} \leqq M_{L, \alpha}<\infty
$$

for any multiindex $\alpha$ and any $L \Subset \Omega$. Assume that we have already proved that $u \in A_{j}^{\sigma}$ for a certain finite $\sigma$ with $0 \leqq \sigma \leqq s$. Choose the same $\chi$ as we did in part (a), then we have

$$
\bar{\partial}(\chi u) \in W_{(p, q+1)}^{\sigma}\left(\boldsymbol{C}^{n}, \phi_{1}\right)
$$

and

$$
\vartheta(\chi u) \in W_{(p, q-1)}^{\sigma}\left(C^{n}, \phi_{1}\right) .
$$

We apply Lemma 4.2 to the above facts. Let $D$ be a differentiation of order $\sigma$. Then

$$
S(D(\chi u))=\bar{\partial}(D(\chi u)) \in L_{(p, q+1)}^{2}\left(C^{n}, \psi_{1}\right)
$$

and

$$
\begin{aligned}
T^{*}(D(\chi u)) & =(-1)^{p-1} e^{\psi_{1}} \vartheta\left(e^{-\psi_{1}}(D \chi u)\right) \\
& =(-1)^{p-1} \vartheta(D(\chi u))+b D(\chi u) \in L_{(p, q-1)}^{2}\left(C^{n}, \psi_{1}\right)
\end{aligned}
$$


where $b$ is a differential oprator of order 0 with bounded $C^{\infty}$ coefficients, and $T^{*}$ is the adjoint operator of $T$ in Lemma 4.2. Since $\psi_{1}$ satisfies the condition in Lemma 4.2 , it follows that $D(\chi u) \in W_{(p, q)}^{1}\left(\boldsymbol{C}^{n}, \psi_{1}\right)$. Hence $\chi u \in W_{(p, q)}^{\sigma+1}\left(C^{n}, \psi_{1}\right)$, that is, $u \in W_{(p, q)}^{\sigma+1}\left(\Omega, \psi_{1}, l o c\right)=A_{j}^{\sigma+1}$. By induction we have $u \in A_{j}^{s}$. This completes the proof.

The following theorem due to $H$. Komatsu is very useful in the later argument.

Theorem 4. 7. Let $X_{1}, X_{2}$ and $X_{3}$ be Fréchet spaces and let $u_{1}: X_{1} \rightarrow X_{2}$ and $u_{2}: X_{2} \rightarrow X_{3}$ be densely defined closed linear mappings such that $u_{2} \circ u_{1}=0$. Denote by $X_{j}^{\prime}$ and $u_{j}^{\prime}$ the strong dual spaces of $X_{j}$ and the dual mappings of $u_{j}$ respectively:

$$
\begin{aligned}
& X_{1} \stackrel{u_{1}}{\longrightarrow} X_{2} \stackrel{u_{2}}{\longrightarrow} X_{3} \\
& X_{1}^{\prime} \stackrel{u_{1}^{\prime}}{\longleftarrow} X_{2}^{\prime} \stackrel{u_{2}^{\prime}}{\longleftarrow} X_{3}^{\prime} .
\end{aligned}
$$

Then:

(i) The image im $u_{j}$ is closed in $X_{j+1}$ if and only if the image im $u_{j}^{\prime}$ is (weakly*) closed in $X_{j}^{\prime}$.

(ii) Suppose that both im $u_{1}$ and im $u_{2}$ are closed. Let $Z=\operatorname{ker} u_{2}$, $B=\operatorname{im} u_{1}, Z^{*}=\operatorname{ker} u_{1}^{\prime}$ and $B^{*}=\operatorname{im} u_{2}^{\prime}$. Then the quotient space $H=Z / B$ is Fréchet and its dual space $H^{\prime}$ is identified with the quotient space $H^{*}=Z^{*} / B^{*}$ as a set. If $X_{2}$ is (FS*) then $H$ is (FS*) and the strong dual space $H^{\prime}$ is isomorphic to $H^{*}$ equipped with the bornologic or the Mackey topology associated with the quotient topology in $H^{*}$. If $X_{2}$ is $(F S)$, then so is $H$ and $H^{\prime}$ is isomorphic to $H^{*}$.

Proof. See Theorem 19 of H. Komatsu [7].

Theorem 4. 8. Let $\Omega$ be a $Q^{k, l}$-pseudoconvex domain in $\boldsymbol{Q}^{k, l}$ and $\bar{\partial}$ be the Cauchy-Riemann operator. Then the sequence

$$
\mathcal{I}_{(p, q-1)}(\Omega) \stackrel{\bar{\partial}}{\rightarrow} \mathcal{I}_{(p, q)}(\Omega) \stackrel{\bar{\partial}}{\rightarrow} \mathcal{I}_{(p, q+1)}(\Omega)
$$

is exact. 
Proof. Let $A_{j}^{s+1}, B_{j}^{s}$, and $C_{j}^{s-1}$ be spaces of differential forms which appeared in Proposition 4.6. We denote by $\left(A_{j}^{s+1}\right)^{\prime},\left(B_{j}^{s}\right)^{\prime},\left(C_{j}^{s-1}\right)^{\prime}$ and $\vartheta$ the dual spaces of $A_{j}^{s+1}, B_{j}^{s}, C_{j}^{s-1}$ and the dual operator of $\bar{\partial}$ respectively. It follows from Proposition 4. 6 that the sequence

$$
A_{j}^{s+1} \stackrel{\bar{\partial}}{\rightarrow} B_{j}^{s} \stackrel{\bar{\partial}}{\rightarrow} C_{j}^{s-1}
$$

is exact. Therefore the sequence

$$
\left(A_{j}^{s+1}\right)^{\prime} \stackrel{\vartheta}{\leftarrow}\left(B_{j}^{s}\right)^{\prime} \Vdash\left(C_{j}^{s-1}\right)^{\prime}
$$

is exact and the ranges of $\vartheta$ are closed by Theorem 4.7. Note that $\left(\mathscr{I}_{(p, q)}(\Omega)\right)^{\prime}=\underline{\lim }\left(B_{j}^{j}\right)^{\prime}$ etc., since $\mathscr{E}(\Omega)=\lim W^{m}(\Omega,|z| / m$, loc $)$ by Proposition 2.25. Since the inductive limit preserves the exactness, we have the exact sequence

$$
\left(\mathscr{F}_{(p, q-1)}(\Omega)\right)^{\prime} \stackrel{\vartheta}{\leftarrow}\left(\mathscr{I}_{(p, q)}(\Omega)\right)^{\prime} \stackrel{\vartheta}{\leftarrow}\left(\mathscr{F}_{(p, q+1)}(\Omega)\right)^{\prime} .
$$

Therefore the theorem is trivially true if $q \geqq 2$ by Theorem 4.7 .

We consider the case $q=1$. By the well-known Krein-Shmulian theorem Fréchet space is fully complete, so we need to prove only $\operatorname{im} \vartheta \cap V^{\circ}$ is closed, where $V$ is a neighbourhood of 0 in $\mathscr{F}_{(p, q-1)}(\Omega)$ and $V^{\circ}$ is the polar set of $V$. Now by Proposition 2.29, there exists some $m$ such that $\operatorname{im} \vartheta \cap V^{\circ}=u_{m}\left(B_{m}\right)$, where $B_{m}$ is a bounded set in $\left[H_{(p, q-1)}^{m+1}\left(\stackrel{\circ}{K}_{m}, \phi_{m}\right)\right]^{\prime}\left(\phi_{m}(z)=\eta(z) / m+4 \log \left(1+|z|^{2}\right)\right)$ and $u_{m}$ is a weak homeomorphism. If we assume $\vartheta u_{\nu} \rightarrow f \in V^{\circ}$, then $\vartheta u_{\nu}$ converges weakly to $f$ in some $\left[H_{(p, q-1)}^{m+1}\left(\stackrel{\circ}{K}_{m}, \phi_{m}\right)\right]^{\prime}$. Here we need the following lemma.

Lemma 4. 9. If $u \in\left[H_{(p, q)}^{k}\left(\stackrel{\circ}{K}_{k}, \psi_{k}\right)\right]^{\prime}\left(\psi_{k}(z)=\eta(z) / k+2 \log \left(1+|z|^{2}\right)\right)$ and $\vartheta u \in\left[H_{(p, q-1)}^{m+1}\left(\stackrel{\circ}{K}_{m}, \phi_{m}\right)\right]^{\prime}(k>m)$, then there exists some $v$ in $\left(B_{m}^{m}\right)^{\prime}$ and $\vartheta u=\vartheta v$ holds.

Proof of the lemma. If we represent $\left[H_{(p, q)}^{k}\left(\stackrel{\circ}{K}_{k}, \psi_{k}\right)\right]^{\prime}$ etc., by the set of $(p, q)$ forms with distribution coefficients $\left[H^{k}\left(\check{K}_{k}, \psi_{k}\right)\right]_{(p, q)}^{\prime}$, then $\vartheta$ is represented by

$$
\vartheta f=(-1)^{p-1} \sum_{I \bar{K}}^{\prime} \sum_{j} \partial f_{I, j K} / \partial \bar{z}_{j} d z^{I} \bigwedge d \bar{z}^{K} .
$$

Let $\phi_{n}(z)=\exp \left(-z^{2} / n\right)$ and $\rho_{\varepsilon} *$ be the Friedrichs mollifier. It is easily 
seen that for $u \in\left[H^{n}\left(\stackrel{\circ}{K}_{k}, \psi_{k}\right)\right]_{(p, q)}^{\prime} \rho_{\varepsilon} * u$ is a $(p, q)$ form with $C^{\infty}$ coefficients whose supports are contained in $\stackrel{\circ}{K}_{k+1} \cap \boldsymbol{C}^{n}$ and $\phi_{n} \rho_{\varepsilon} * u$ belongs to $\left[H^{m}\left(\stackrel{\circ}{K}_{k+1}, \psi_{m}\right)\right]_{(p, q)}^{\prime}$ since we have assumed

$$
\sup _{z \subseteq \Omega \cap \boldsymbol{C}^{n}, 1 \leq i \leq j}\left\{2\left|\operatorname{Im} \underline{z}_{k_{i}}\right|^{2}-\left|\operatorname{Re} z_{i}^{\prime}\right|^{2},\left|\operatorname{Im} \underline{z}_{l_{i}}\right|^{2}\right\}<\infty .
$$

Thus $\phi_{n} \rho_{\varepsilon} * u$ belongs to

$$
\left(B_{m}^{m}\right)^{\prime}=\left[W_{(p, q)}^{m}\left(\Omega, \phi_{m}, \mathrm{loc}\right)\right]^{\prime}=\lim _{k}\left[H^{m}\left(\stackrel{\circ}{K}_{k}, \phi_{m}\right)\right]_{(p, q)}^{\prime} .
$$

Note that $\vartheta \phi_{n} \rho_{\varepsilon} * u=\phi_{n} \vartheta \rho_{\varepsilon} * u=\phi_{n} \rho_{\varepsilon} *(\vartheta u)$ and $\phi_{n} \rho_{\varepsilon} *(\vartheta u)$ converges to $\vartheta u$ in $\left[W_{(p, q-1)}^{m+1}\left(\Omega, \phi_{m}, \text { loc }\right)\right]^{\prime}=\left(A_{m}^{m+1}\right)^{\prime}$ as $n \rightarrow \infty$ and $\varepsilon \rightarrow 0$. Since $\vartheta$ is a closed range operator from $\left(B_{m}^{m}\right)^{\prime}$ to $\left(A_{m}^{m+1}\right)^{\prime}$, there exists some $v$ in $\left(B_{m}^{m}\right)^{\prime}$ such that $\vartheta v=\vartheta u$.

We return to the prooof of Theorem 4.8. By Lemma 4.9 we may assume not only $\vartheta u_{\nu} \in\left(A_{m}^{m+1}\right)^{\prime}$ but also $u_{\nu} \in\left(B_{m}^{m}\right)^{\prime}$. Since $\vartheta u_{\nu}$ converges weakly to $f$ in $\left[H^{m+1}\left(\stackrel{\circ}{K}_{m}, \phi_{m}\right)\right]_{(p, q-1)}^{\prime}, \vartheta u_{\nu}$ converges weakly to $f$ in

$$
\left(A_{m}^{m+1}\right)^{\prime}=\lim _{j}\left[H^{m+1}\left(\stackrel{\circ}{K}_{j}, \phi_{m}\right)\right]_{(p, q)}^{\prime} .
$$

Since $\vartheta\left(\mathrm{B}_{m}^{m}\right)^{\prime}$ is closed in $\left(A_{m}^{m+1}\right)^{\prime}, \vartheta\left(B_{m}^{m}\right)^{\prime}$ is weakly closed because $\vartheta\left(B_{m}^{m}\right)^{\prime}$ is convex. Therefore there exists some $v$ in $\left(B_{m}^{m}\right)^{\prime}$ such that $f=\vartheta v$. This proves that $\operatorname{im} \vartheta \cap V^{\circ}$ is closed, hence $\operatorname{im} \vartheta$ is closed. Then it follows from Theorem 4. 7 that the sequence (4.3) is exact.

For the later use, we prepare the following proposition.

Proposition 4. 10. Let $\Omega$ be a pseudoconvex domain in $C^{n}$ and $\psi(z)$ be a plurisubharmonic function in $\Omega$. We define $X=\lim X_{j}$, $Y=\lim Y_{j}$ and $Z=\lim Z_{j}$ where $X_{j}, Y_{j}$ and $Z_{j}$ are those spaces which appeared in Lemma 4. 4. Then we have the following exact sequence:

$$
X \stackrel{\bar{\partial}}{\rightarrow} Y \stackrel{\bar{\partial}}{\rightarrow} Z
$$

Proof. See Lemma 2.1.1 of T. Kawai [6].

Now we will show that every point $z$ in the open subset $\Omega$ in $Q^{k, l}$ which satisfies the condition (i) of Definition 4.5, has a fundamental system of $Q^{k, l}$-pseudoconvex domains. If $z$ belongs to $\boldsymbol{C}^{n}$, it is evident that 
$z$ has a fundamental system of $Q^{k, l}$-pseudoconvex domains, since the relatively compact convex open set in $\boldsymbol{C}^{n}$ is a pseudoconvex domain, hence it is a $Q^{k, l}$-pseudoconvex domain. Then we consider the case that $z_{\infty}$ belongs to $\prod_{i=1}^{j}\left(\boldsymbol{S}_{\infty}^{2 k_{i}+l_{i}-1} \times \sqrt{-1} \boldsymbol{R}^{i_{i}}\right)$. First, let $z^{(1)}=\left(e_{1}, \cdots, e_{j}\right)+\sqrt{-1} 0$, where $e_{i}=(1,0, \cdots, 0) \in \boldsymbol{S}^{2 k_{i}+l_{i}-1}$ and $z_{\infty}^{(1)}$ be the point at infinity corresponding to $z^{(1)}$. We define $p_{k, l}^{\varepsilon}(z)=\gamma_{k, l}^{\varepsilon}(z)+\sum_{j=k+1}^{k+l}\left|\operatorname{Im} z_{j}\right|^{2}$ for $z \in C^{n}$ and positive integers $k, l$, where

$$
\gamma_{k, l}^{\varepsilon}(z)=\left(\left|\operatorname{Im} z_{1}\right|^{2}+\sum_{j=2}^{k+l}\left|z_{j}\right|^{2}\right) /\left|\operatorname{Re} z_{1}-1 / \varepsilon\right|^{2}
$$

and

$$
p^{\varepsilon}(z)=\sum_{i=1}^{j} p_{k_{i}, l_{i}}^{\varepsilon}\left(z_{i}^{\prime}\right)
$$

for $z=\left(z_{1}^{\prime}, \cdots, z_{j}^{\prime}\right) \in \prod_{i=1}^{j}\left(\boldsymbol{Q}^{k_{i}, l_{i}} \cap \boldsymbol{C}^{n_{i}}\right)=\boldsymbol{Q}^{k, \imath} \cap \boldsymbol{C}^{n}\left(k_{i}+l_{i}=n_{i}, \sum_{i=1}^{j} n_{i}=n\right)$.

Let $U_{\varepsilon}=\left\{z \in C^{n} ; p^{\varepsilon}(z)<\varepsilon\right\}$ and $V_{\varepsilon}=\stackrel{\circ}{\bar{U}}_{\varepsilon}$, then $\left\{V_{\varepsilon}\right\}_{0<\varepsilon<1 / 4}$ is a fundamental system of neighbourhoods of $z_{\infty}^{(1)}$ which consists of $Q^{k, l}$-pseudoconvex domains. To show this, it is sufficient to prove that $q^{\varepsilon}(z)$ $=1 /\left(p^{\varepsilon}(z)-\varepsilon\right)$ satisfies the condition (ii) of Definition 4.5. In fact, it can be easily seen that $q^{\varepsilon}(z)$ is $C^{\infty}$ strictly plurisubharmonic in $U_{\varepsilon}$ and the derivatives of $p^{\varepsilon}(z)$ are all bounded on $K \cap C^{n}$ for any compact set $K$ in $V_{\varepsilon}$. Thus we see that $q^{\varepsilon}(z)$ satisfies the required condition. For the general $z_{\infty}=z_{\infty}^{\prime}+i y \in \prod_{i=1}^{j}\left(S_{\infty}^{2 k_{i}+l_{i}-1} \times \sqrt{-1} \boldsymbol{R}^{l_{i}}\right)$, we choose a transformation $T$ which is a tensor product of unitary operators $T_{i}$ in $C^{n_{i}}$, that is $T=\bigotimes_{i=1}^{j} T_{i}$ satisfying $T\left(z_{\infty}^{\prime}\right)=z_{\infty}^{(1)}$. Let

$$
p^{\varepsilon}(z)=\sum_{i=1}^{j}\left\{\gamma_{k_{i}, l_{i}}^{\varepsilon}\left(T_{i}\left(z_{i}^{\prime}\right)\right)+\left|\operatorname{Im} \underline{z}_{l_{i}}-\underline{y}_{l_{i}}\right|^{2}\right\}
$$

then $V_{\varepsilon}=\stackrel{\circ}{\bar{U}}_{\varepsilon}$ for $U_{\varepsilon}=\left\{z \in \boldsymbol{C}^{n} ; p^{\varepsilon}(z)<\varepsilon\right\}, \quad 0<\varepsilon<\rho_{z_{\infty}}$ is a $Q^{k, l}$-pseudoconvex neighbourhood of $z_{\infty}$, where $\rho_{z_{\infty}}$ is an upper bound of $\varepsilon$ in order that $U_{\varepsilon}$ satisfies the condition (i) of Definition 4.5.

Now, let $\mathscr{F}_{(0, p)}$ be the sheaf subordinated to the presheaf $\left\{\mathscr{F}_{(0, p)}(\Omega)\right\}$, then it is easy to see that the sheaf is soft. From Theorem 4.8, we 
have the following theorem, which is the key theorem to construct vector valued Fourier hyperfunctions.

Theorem 4. 11. Let $\Omega$ be an open set in $Q^{k, l}$ satisfying the condition (i) of Definition 4.5. We have the following soft resolution of the sheaf $\widetilde{\mathcal{O}}_{k, l}$ on $\Omega$ :

$$
0 \rightarrow \widetilde{\mathcal{O}}_{k, l} \rightarrow \mathscr{I}_{(0,0)} \stackrel{\bar{\partial}}{\rightarrow} \mathscr{I}_{(0,1)} \stackrel{\bar{\partial}}{\rightarrow} \cdots \stackrel{\bar{\partial}}{\rightarrow} \mathscr{I}_{(0, n)} \rightarrow 0 .
$$

Therefore the cohomology group $H^{p}\left(\Omega, \widetilde{\mathcal{O}}_{k, l}\right)$ is isomorphic to the p-th cohomology group of the following complex:

$$
0 \rightarrow \mathscr{F}_{(0,0)}(\Omega) \stackrel{\bar{\partial}}{\rightarrow} \mathscr{I}_{(0,1)}(\Omega) \stackrel{\bar{\partial}}{\rightarrow} \cdots \stackrel{\bar{\partial}}{\rightarrow} \mathscr{F}_{(0, n)}(\Omega) \rightarrow 0 .
$$

\section{§ 5. Vanishing Theorems and Duality Theorems of Cohomology Groups}

In this section, we use the resolution of $\widetilde{\mathcal{O}}_{k, l}$ by the projective limit of $L^{2}$ spaces which is convenient to give duality theorems. This resolution was used by T. Kawai [6] in type I case and by Y. Saburi [13], [14] in type II case. In the case of mixed type, we can also obtain the $L^{2}$ resolution of $\widetilde{\mathcal{O}}_{k, l}$ and calculate the cohomology groups in the same way.

In this section we use the following definition of $\widetilde{\mathcal{O}}_{k, l}$-pseudoconvexity which was used in T. Kawai [6] and Y. Saburi [13], [14], and is weaker than Definition 4. 5 .

Definition 5. 1. We call an open set $\boldsymbol{\Omega}$ in $\boldsymbol{Q}^{k, l}$ an $\widetilde{\mathcal{O}}_{k, l}$-pseudoconvex domain if it satisfies the following conditions:

(i) $\sup _{z \in \mathscr{S} \cap \boldsymbol{C}^{n}, 1 \leq i \leq j}\left\{2\left|\operatorname{Im} \underline{z}_{k_{i}}\right|^{2}-\left|\operatorname{Re} z_{i}^{\prime}\right|^{2},\left|\operatorname{Im} \underline{z}_{l_{i}}\right|^{2}\right\} \leqq M<\infty, \quad$ where $\quad z_{i}^{\prime}$ $=\left(\underline{z}_{k_{i}}, \underline{z}_{l_{i}}\right) \in \boldsymbol{Q}^{k_{i}, l_{i}}$ and $\boldsymbol{Q}^{k, l}=\prod_{i=1}^{j} \boldsymbol{Q}^{k_{i}, l_{i}}$.

(ii) There exists a $C^{\infty}$ strictly plurisubharmonic function $\theta(z)$ on $\Omega \cap C^{n}$ which satisfies $\{z ; \theta(z)<c\} \Subset \Omega$ for any real $c$ and $\sup _{z \in L \cap \boldsymbol{C}^{n}}|\theta(z)|$ $\leqq M_{L}$ for any $L \subset \Omega$, where $M_{L}$ is some constant. 
Remark 5.2. In the case of $L^{2}$ resolution of $\widetilde{\mathcal{O}}_{k, l}$, it is not neccessary to assume that the derivative of $\theta(z)$ are bounded, which is assumed in Definition 4.5.

Theorem 5. 3. Let $S$ be an open set in $\prod_{i=1}^{j} \boldsymbol{D}^{n_{i}}$ and $U$ be an open neighbourhood of $S$ in $\boldsymbol{Q}^{k, l}$, then there exists an $\widetilde{\mathcal{O}}_{k, l}$-pseudoconvex domain $V$ which satisfies the condition: $V \subset U$ and $S=V \cap\left(\prod_{i=1}^{j} \boldsymbol{D}^{n_{i}}\right)$.

Proof. For the type I and type II cases, this theorem has been proved by T. Kawai [6] and Y. Saburi [13] respectively. For the mixed type we can prove it in a way similar to T. Kawai [6] and Y. Saburi [13]. We have only to mix their methods.

First, choose $\gamma(z) \in C^{\infty}\left(U \cap \boldsymbol{C}^{n}\right)$ which satisfies

(i) $\left\{z \in U \cap \boldsymbol{C}^{n} ; \gamma(z) \leqq c\right\} \Subset U$ for any $c$,

(ii) $\sup _{L \cap \boldsymbol{C}^{n}}\left\{|\gamma(z)|,(1+|z|)|\nabla \gamma(z)|,(1+|z|)^{2}\left|\nabla^{2} \gamma(z)\right|\right\} \leqq M_{L}<\infty \quad$ for $L \mathbb{E} U$,

where $\nabla$ means any of $\partial / \partial x_{i}$ or $\partial / \partial y_{j}$ and $\nabla^{2}$ means any of $\partial^{2} / \partial x_{i} \partial x_{j}$, $\partial^{2} / \partial x_{i} \partial y_{j}$ or $\partial^{2} / \partial y_{i} \partial y_{j}$. Next choose $a(x) \in C^{\infty}\left(S \cap \boldsymbol{R}^{n}\right)$ which grows sufficiently rapidly as $x$ tends to the boundary of $\left(\boldsymbol{D}^{n}-S\right)$ from the interior of $S \cap \boldsymbol{R}^{n}$, but $\sup _{\text {an }}\left\{|a(x)|,(1+|x|)|\nabla a(x)|,(1+|x|)^{2}\left|\nabla^{2} a(x)\right|\right\}$ $\leqq N_{K}<\infty$ for any $K \Subset S$. Define $p(z)=a(\operatorname{Re} z)|\operatorname{Im} z|^{2} /\left(1+\left|\operatorname{Re} \underline{z}_{k}\right|^{2}\right)$ $+\gamma(z)\left(z=\left(\underline{z}_{k}, \underline{z}_{l}\right)\right)$. Then we can fined a neighbourhood $W(\subset U)$ of $S$ in $\boldsymbol{Q}^{k, l}$ such that $p(z)$ is strictly plurisubharmonic in $W$. For the construction of $\gamma(z)$ and $a(x)$ and the detailed proof of the plurisubharmonicity of $p(z)$, see Y. Saburi [13].

Now, let us define

$$
g_{i}(z)=\max \left\{0,\left[2|\operatorname{Im} z|^{2}-\left|\operatorname{Re}\left(z-z^{(i)}\right)\right|^{2}\right] /\left|\operatorname{Im} z^{(i)}\right|^{2}\right\}
$$

and $\chi(x) \in C^{\infty}(\boldsymbol{R})$ to be a convex monotone increasing function such that $\chi(x)=0$ if $x \leqq 0$ and $\chi(x)>0$ if $x>0$. By a suitable choice of $z^{(i)}$ $\in\left(\partial W-\boldsymbol{D}^{n}\right) \cap \boldsymbol{C}^{n}$, we may assume that the sum $q(z)=\sum_{i} \chi\left(g_{i}(z)\right)$ is locally finite in $\boldsymbol{C}^{n}$ and $q(z)>1$ if $z \in\left(\partial W-\boldsymbol{D}^{n}\right) \cap \boldsymbol{C}^{n}$. Then the domain $V=W \cap \frac{o}{\left\{z \in C^{n} ; q(z)<1\right\}}$ is an $\widetilde{\mathcal{O}}_{k, \imath}$-pseudoconvex domain with a plurisubharmonic function $\theta(z)=p(z)+1 /(1-q(z))$. 
Definition 5. 4. Let $\Omega$ be an open set in $\boldsymbol{Q}^{k, l}$. We define $\mathscr{X}_{j}(\Omega)$ to be the set of all $(0, j)$ forms $u$ on $\Omega \cap C^{n}$ which satisfy the following conditions: For any compact set $K$ in $\Omega$ and any positive $\varepsilon$,

$$
\int_{K \cap \boldsymbol{C}^{n}}|u|^{2} e^{-\varepsilon_{\eta}(z)} d \lambda<\infty \text { and } \int_{K \cap \boldsymbol{C}^{n}}|\bar{\partial} u|^{2} e^{-\varepsilon_{\eta}(z)} d \lambda<\infty
$$

hold. We denote by $\mathscr{X}_{j}$ the sheaf subordinate to the presheaf $\left\{\mathscr{X}_{j}(\Omega)\right\}$.

Definition 5. 5. Let $\Omega$ be an open set in $\boldsymbol{Q}^{k, l}$. We define $\mathcal{G}_{j}(\Omega)$ to be the set of all $(0, j)$ forms $u$ on $\Omega \cap C^{n}$ which satisfy the following conditions: For any compact set $K$ in $\Omega$, there exists some positive $\delta_{K}$ such that

$$
\int_{K \cap \boldsymbol{C}^{n}}|u|^{2} e^{\delta_{K \eta}(z)} d \lambda<\infty \text { and } \int_{K \cap \boldsymbol{C}^{n}}|\bar{\partial} u|^{2} e^{\delta_{K \eta}(z)} d \lambda<\infty
$$

hold. We denote by $\mathscr{f}_{j}$ the sheaf subordinate to the presheaf $\left\{\mathscr{y}_{j}(\Omega)\right\}$.

It is easily seen that the sheaves $\mathscr{X}_{j}$ and $\mathscr{Y}_{j}$ are soft sheaves. By the definition of $\widetilde{\mathcal{O}}_{k, l}$ and $\mathcal{Q}_{k, l}$ and the existence theorem for $\bar{\partial} u=f$ with bounds (Proposition 4.10) we obtain the soft resolutions of $\widetilde{\mathcal{O}}_{k, l}$ and $\mathcal{Q}_{k, l}$ respectively

$$
\begin{aligned}
& 0 \rightarrow \widetilde{\mathcal{O}}_{k, l} \rightarrow \mathscr{X}_{0} \rightarrow \cdots \rightarrow \mathscr{X}_{n} \rightarrow 0 \\
& 0 \rightarrow \mathcal{O}_{k, l} \rightarrow \mathscr{Y}_{0} \rightarrow \cdots \rightarrow \mathscr{Y}_{n} \rightarrow 0 .
\end{aligned}
$$

Therefore we obtain the following Dolbeault isomorphism:

$$
\begin{aligned}
& H^{p}\left(\Omega, \widetilde{\mathcal{O}}_{k, l}\right)=\left\{u \in \mathscr{X}_{p}(\Omega) ; \bar{\partial} u=0\right\} / \bar{\partial} \mathscr{X}_{p-1}(\Omega) \\
& H_{c}^{p}\left(\Omega, \mathcal{O}_{k, l}\right)=\left\{u \in \mathcal{Y}_{p}(\Omega)_{c} ; \bar{\partial} u=0\right\} / \bar{\partial} \mathcal{Y}_{p-1}(\Omega)_{c} .
\end{aligned}
$$

Here we denote by $H_{c}^{p}\left(\Omega, \mathcal{Q}_{k, l}\right)$ the $p$-th cohomology groups with compact support. In order to compute the above cohomology groups we introduce the following spaces.

Definition 5. 6. Let $\Omega$ be an open set in $\boldsymbol{Q}^{k, l}$. We denote by $X_{j}(\Omega)$ the set of all $(0, j)$ forms $u$ on $\Omega \cap C^{n}$ satisfying the following condition: For any compact set $K$ in $\Omega$ and any positive $\varepsilon$,

$$
\int_{K \cap \boldsymbol{C}^{n}}|u|^{2} e^{-\varepsilon_{\eta}(z)} d \lambda<\infty
$$


holds.

Definition 5. 7. Let $\Omega$ be an open set in $\boldsymbol{Q}^{k, l}$. We denote by $Y_{j}(\Omega)$ the set of $(0, j)$ forms $u$ with compact support in $\Omega$ satisfying the condition: For some positive $\delta$,

$$
\int_{\boldsymbol{C}^{n}}|u|^{2} e^{\delta \eta(z)} d \lambda<\infty
$$

holds.

From the above definition $X_{j}(\Omega)$ and $Y_{j}(\Omega)$ are given natural $F S^{*}$ and $D F S^{*}$ space structures respectively, that is,

$$
\begin{aligned}
& X_{j}(\Omega)=\lim _{m} L_{(0, j)}^{2}\left(\stackrel{\circ}{K}_{m}, \eta(z) / m\right), \\
& Y_{j}(\Omega)={\stackrel{\lim }{m} L_{(0, j)}^{2}\left(\stackrel{\circ}{K}_{m},-\eta(z) / m\right)}^{\longleftrightarrow},
\end{aligned}
$$

and using the natural identification of $(0, j)$ form with $(0, n-j)$ form, we have $Y_{n-j}(\Omega)=\left[X_{j}(\Omega)\right]^{\prime}$ where $K_{1} \Subset K_{2} \subset \cdots$ is the increasing sequence of compact sets in $\Omega$ which exhaust $\Omega$.

Then $H^{p}\left(\Omega, \widetilde{O}_{k, l}\right)$ is isomorphic to the $p$-th cohomology group of the complex

$$
X_{p-1}(\Omega) \stackrel{\bar{\partial}}{\rightarrow} X_{p}(\Omega) \stackrel{\bar{\partial}}{\rightarrow} X_{p+1}(\Omega)
$$

and $H_{c}^{q}\left(\Omega, \mathcal{O}_{k, l}\right)$ is isomorphic to the $q$-th cohomology group of the complex

$$
Y_{q+1}(\Omega) \stackrel{-\bar{\partial}}{\longleftarrow} Y_{q}(\Omega) \stackrel{-\bar{\partial}}{\longleftarrow} Y_{q-1}(\Omega) .
$$

Therefore we obtain the following theorem from Theorem 4. 7, since $-\bar{\partial}$ is the dual operator of $\bar{\partial}$.

Theorem 5. 8. If $H^{p}\left(\Omega, \widetilde{\mathcal{O}}_{k, l}\right)=0(p \geqq 1)$ then $\left[H^{j}\left(\Omega, \widetilde{\mathcal{O}}_{k, l}\right)\right]^{\prime}$ $=H_{c}^{n-j}\left(\Omega, \mathcal{O}_{k, l}\right)$.

Concerning to the hypothesis of the above theorem, we have the following theorem. 
Theorem 5.9. For any $\widetilde{\mathcal{O}}_{k, l}$-pseudoconvex domain $\Omega$, we have $H^{p}\left(\Omega, \widetilde{\mathcal{O}}_{k, l}\right)=0 \quad(p \geqq 1)$.

Proof. From Proposition 4.10 and the existence of the plurisubharmonic function $\theta(z)$ satisfying the condition (i) and (ii) of Definition 5. 1 , follows the theorem.

Corollary 5. 10. Let $K$ be a compact set in $\boldsymbol{Q}^{k, l}$ which has a fundamental system of neighbourhoods composed of $\widetilde{\mathcal{O}}_{k, l}$-pseudoconvex domains $\Omega_{j}$, then

$$
H^{p}\left(K, \mathcal{Q}_{k, l}\right)=0 \quad(p \geqq 1) \text {. }
$$

Proof. It is sufficient to show that

$$
\lim _{j} H^{p}\left(\Omega_{j},{\underset{Q}{k, l}}_{l}\right)=0 \quad(p \geqq 1) .
$$

Let $u(z)$ be arbitrary cocycle in $H^{p}\left(\Omega_{j},{\underset{\sim}{k, l}}_{k}\right)$. Then $u(z)$ cosh $\varepsilon z$ belongs to $H^{p}\left(\Omega_{j+1}, \mathcal{O}_{k, l}\right)$ for sufficiently small $\varepsilon>0$. By Theorem $5.9 u(z)$ $\times \cosh \varepsilon z$ is a coboundary in $H^{p}\left(\Omega_{j+1}, \widetilde{\mathcal{O}}_{k, l}\right)$, that is, $u(z) \cosh \varepsilon z=\bar{\partial} v(z)$ for some $v(z) \in \mathfrak{X}_{p-1}\left(\Omega_{j+1}\right)$. Define $w(z)=v(z) / \cosh \varepsilon z$. For sufficiently small $\varepsilon>0, w(z)$ belongs to $\mathcal{Y}_{p-1}\left(\Omega_{j+1}\right)$ and $\bar{\partial} w(z)=u(z)$, hence the image of $H^{p}\left(\Omega_{j}, \mathcal{\sim}_{k, l}\right)$ in $H^{p}\left(\Omega_{j+1},{\underset{\sim}{k, l}}_{k}\right)$ is zero. Therefore $\lim _{j} H^{p}\left(\Omega_{j}, \mathcal{Q}_{k, l}\right)$ $=H^{p}\left(K, \mathcal{O}_{k, l}\right)=0$.

Theorem 5. 11. Let $\Omega$ be any open set in $Q^{k, l}$ satisfying $\sup _{z \in \Omega \cap \boldsymbol{C}^{n}, 1 \leqq i \leqq j}\left\{2\left|\operatorname{Im} \underline{z}_{k_{i}}\right|^{2}-\left|\operatorname{Re} z_{i}^{\prime}\right|^{2},\left|\operatorname{Im} \underline{z}_{l_{i}}\right|^{2}\right\} \leqq M<\infty$, then $H^{n}\left(\Omega, \widetilde{\mathscr{O}}_{k, l}\right)=0$.

Proof. We can prove the theorem in the same way as Theorem 3. 1.8 in T. Kawai [6].

Using above theorems, we have the following theorem.

Theorem 5. 12. Let $K$ be a compact set in $Q^{k, l}$, and $V$ be an $\widetilde{\mathcal{O}}_{k, l}$-pseudoconvex domain containing $K$. Suppose $H^{p}\left(K, \mathcal{O}_{k, l}\right)=0$ for $p \geqq 1$. Then we have $H_{K}^{p}\left(V, \widetilde{\mathcal{O}}_{k, l}\right)=0(p \neq n)$ and $H_{K}^{n}\left(V, \widetilde{\mathcal{O}}_{k, l}\right)=\left[\mathcal{Q}_{k, l}(K)\right]^{\prime}$. 
Proof. We have only to replace $\widetilde{\mathcal{O}}$ by $\widetilde{\mathcal{O}}_{k, l}$ and $\mathcal{O}$ by $\mathcal{O}_{k, l}$ in the proof of Theorem 3.2.1 of T. Kawai [6].

If $K$ is a compact set in $\prod_{i=1}^{j} D^{n_{i}}, K$ has a fundamental system of neighbourhoods composed of $\widetilde{\mathcal{O}}_{k, l}^{i=1}$-pseudoconvex domains by Theorem 5. 3 . Thus we have the following theorem.

Theorem 5. 13. Let $K$ be a compact set in $\prod_{i=1}^{j} \boldsymbol{D}^{n_{i}}$ and $V$ be an open neighbourhood of $K$ in $\boldsymbol{Q}^{k, l}$. Then we have $H_{K}^{p}\left(V, \widetilde{\mathcal{O}}_{k, l}\right)=0$ $(p \neq n)$ and $H_{K}^{n}\left(V, \widetilde{\mathcal{O}}_{k, l}\right)=\left[\mathcal{\Theta}_{k, l}(K)\right]^{\prime}$.

As is well known, this theorem combined with Theorem 5.11, concludes the pure-codimentionality of $\widetilde{\mathcal{O}}_{k, l}$ with respect to $\prod_{i=1}^{j} \boldsymbol{D}^{n_{i}}$, or the following theorem holds.

Theorem 5. 14. Let $\Omega$ be an open set in $\prod_{i=1}^{j} \boldsymbol{D}^{n_{i}}$. Then $H_{a}^{p}\left(V, \widetilde{\mathcal{O}}_{k, l}\right)$ $=0(p \neq n)$, where $V$ is an open set in $\boldsymbol{Q}^{k, l}$ containing $\Omega$ as a rela tively closed set.

\section{§ 6. Vector Valued Fourier Hyperfunctions}

In this section, we define Fourier hyperfunctions which take their values in a Fréchet space $E$, and we call them $E$-valued Fourier hyperfunctions. In the case where $E$ is the one dimensional space of complex numbers, $E$-valued Fourier hyperfunctions are ordinary Fourier hyperfunctions.

Definition 6. 1. Let $\Omega$ be an open set in $Q^{k, l}$ and $E$ be a Fréchet space. We denote by $\mathscr{E}(\Omega, E)$ the set of $E$-valued $C^{\infty}$ functions on $\Omega \cap \boldsymbol{C}^{n}$ which satisfy the following condition:

For any compact set $K$ in $\Omega$, any positive $\varepsilon$ and integer $m$ and any continuous seminorm $p$ on $E$,

$$
\sup _{z \in K \cap \boldsymbol{C}^{n},|\alpha| \leqq m} p\left(D^{\alpha} f(x, y)\right) e^{-\varepsilon|z|}<\infty
$$

where $z=x+i y$. 
Proposition 6. 2. Let $\Omega$ be an open set in $\boldsymbol{Q}^{k, l} . \quad C_{0}^{\infty}\left(\Omega \cap C^{n}\right) \otimes E$ is dense in $\mathscr{I}(\Omega, E)$.

Proof. Let $f \in \mathscr{F}(\Omega, E)$. Consider an increasing sequence $K_{1} \subset K_{2}$ $\Subset \cdots$ of compact sets in $\Omega$ which exhaust $\Omega$ and $C^{\infty}$ functions $\phi_{m}(z)$ on $\boldsymbol{C}^{n}$ whose support are contained in $K_{m} \cap \boldsymbol{C}^{n}$ and $\phi_{m}(z)=1$ if $z \in K_{m-1} \cap \boldsymbol{C}^{n}$, then $\phi_{m} f \in \mathscr{F}_{c}\left(K_{m}, E\right)$ and $\phi_{m} f \rightarrow f$ in $\mathscr{I}(\Omega, E)$ as $m$ tends to infinity. Let $\eta_{\nu}(z)$ be $C^{\infty}$ functions with compact support in $C^{n}$ such that $\eta_{\nu}(z)=1$ if $|z| \leqq \nu$ and their derivatives are bounded uniformaly in $\nu$, then $\eta_{\nu} \phi_{m} f \rightarrow \phi_{m} f$ in $\mathscr{E}(\Omega, E)$ as $\nu$ tends to infinity. Thus $\eta_{m} \phi_{m} f \rightarrow f$ in $\mathscr{F}(\Omega, E)$ and $\eta_{m} \phi_{m} f \in C_{0}^{\infty}\left(\Omega \cap C^{n}, E\right)$.

Let $g \in C_{0}^{\infty}\left(\Omega \cap C^{n}, E\right)$ and $p$ be a continuous seminorm of $E$. For any positive $\varepsilon$ we can find a finite covering $U_{1}, \cdots, U_{r}$ of the support of $g$ by relatively compact open subsets of $\Omega \cap \boldsymbol{C}^{n}$ such that $p(g(z)-g(y))$ $<\varepsilon$ for $y, z \in U_{j}(j=1, \cdots, r)$. Let $\left\{h_{j}\right\}$ be a $C^{\infty}$ partition of unity on the support of $g$, subordinated to the above covering, and in each set $U_{j}$, pick up a point $y_{j}$. Then we have for any positive integer $m$,

$$
\begin{aligned}
p(g(z) & \left.-\sum h_{j}(z) g\left(y_{j}\right)\right) e^{-|z| / m} \\
& \leqq \sum h_{j}(z) p\left(g(z)-g\left(y_{j}\right)\right) e^{-|z| / m}<\varepsilon .
\end{aligned}
$$

This shows that $g \in C_{0}^{\infty}\left(\Omega \cap C^{n}, E\right)$ is a limit of $g_{j} \in C_{0}^{\infty}\left(\Omega \cap C^{n}\right) \otimes E$ in the topology defined by the seminorms

$$
p_{K, m}(f)=\sup _{z \in K \cap \boldsymbol{C}^{n}} p(f(z)) e^{-|z| / m},
$$

where $K$ is a compact set in $\Omega$ and $m$ is a positive integer. Let $\rho_{\varepsilon} *$ be the usual Friedrichs mollifier. Then it is not difficult to see that for a sufficiently small $\varepsilon>0, \rho_{\varepsilon} * g$ is the limit of $\rho_{\varepsilon} * g_{j}$ in $\mathscr{I}(\Omega, E)$, since we may assume that the supports of $g_{j}$ are contained in an arbitrary neighbourhood $U$ of the support of $g$.

Finally we see that $\rho_{\varepsilon} * g_{j} \in C_{0}^{\infty}\left(\Omega \cap C^{n}\right) \otimes E$ converges to $\rho_{\varepsilon} * g$ $\left(\in C_{0}^{\infty}\left(\Omega \cap \boldsymbol{C}^{n}, E\right)\right)$ in $\mathscr{F}(\Omega, E)$ and $\rho_{\varepsilon} * g$ converges to $g\left(\in C_{0}^{\infty}\left(\Omega \cap C^{n}, E\right)\right.$ ) in $\mathscr{I}(\Omega, E)$, but there is a sequence $f_{j} \in C_{0}^{\infty}\left(\Omega \cap C^{n}, E\right)$ which converges to $f \in \mathscr{F}(\Omega, E)$ in the topology in $\mathscr{I}(\Omega, E)$. This completes the proof.

Proposition 6. 3. $\mathscr{I}(\Omega, E)$ is complete. 
Proof. Let $\left\{f_{j}\right\}$ be a Cauchy sequence in $\mathscr{I}(\Omega, E)$. As $E$ is complete, $f_{j}$ converges pointwise to a function $f$, moreover $f_{j}$ converges to $f$ in the topology defined by the seminorms

$$
p_{K, m}(f)=\sup _{z \in K \cap \boldsymbol{C}^{n}} p(f(z)) e^{-|z| / m} .
$$

Therefore $f(z)$ is continuous function in $\Omega \cap C^{n}$ satisfying $p_{K, m}(f)<\infty$. In the same way $D^{\alpha} f_{j}$ converges to a continuous function $f_{\alpha}$ satisfying $p_{K, m}\left(f_{\alpha}\right)<\infty$. Then we have only to show that $D^{\alpha} f=f_{\alpha}$. Let $\alpha=\left(\alpha_{1}, \cdots, \alpha_{2 n}\right)$ with $\alpha_{p+1}=\alpha_{p+2}=\cdots=\alpha_{2 n}=0$. Then we have

$$
\begin{aligned}
f_{j}(z)-f_{j}(\zeta)= & \int_{\xi_{1}}^{x_{1}} \cdots \int_{\xi_{p}}^{x_{p}} \frac{\left(x_{1}-y_{1}\right)^{\alpha_{1}-1} \cdots\left(x_{p}-y_{p}\right)^{\alpha_{p}-1}}{\left(\alpha_{1}-1\right) ! \cdots\left(\alpha_{p}-1\right) !} \\
& \times D^{\alpha} f_{j}\left(y_{1}, \cdots, y_{p}, x_{p+1}, \cdots, x_{2 n}\right) d y_{1} \cdots d y_{p},
\end{aligned}
$$

where we put $z=\left(x_{1}, \cdots, x_{2 n}\right), \zeta=\left(\xi_{1}, \cdots, \xi_{2 n}\right)$ and $x_{j}=\xi_{j}$ for $p+1 \leqq j$ $\leqq 2 n$. Since $f_{j}$ and $D^{\alpha} f_{j}$ converge uniformly to $f$ and $f_{\alpha}$ respectively on every compact set $L$ in $\Omega \cap C^{n}$, we have

$$
\begin{aligned}
f(z)-f(\zeta)= & \int_{\xi_{1}}^{x_{1}} \cdots \int_{\xi_{p}}^{x_{p}} \frac{\left(x_{1}-y_{1}\right)^{\alpha_{1}-1} \cdots\left(x_{p}-y_{p}\right)^{\alpha_{p}-1}}{\left(\alpha_{1}-1\right) ! \cdots\left(\alpha_{p}-1\right) !} \\
& \times f_{\alpha}\left(y_{1}, \cdots, y_{p}, x_{p+1}, \cdots, x_{2 n}\right) d y_{1} \cdots d y_{p} .
\end{aligned}
$$

Thus we have $D^{\alpha} f=f_{\alpha}$.

Proposition 6. 4. $\mathscr{F}(\Omega, E)=\mathscr{F}(\Omega) \hat{\otimes}_{\varepsilon} E$.

Proof. By Propositions 6. 2 and 6. 3, it suffices to show that $\mathscr{I}(\Omega, E)$ induces on $\mathscr{I}(\Omega) \otimes E$ the topology $\varepsilon$.

We observe, first, that $\mathscr{F}(\Omega, E)$ can be canonically injected in $L\left(E_{\tau}^{\prime} ; \mathscr{F}(\Omega)\right)$ the space of continuous linear mapping from $E_{\tau}^{\prime}$ to $\mathscr{I}(\Omega)$, where the index $\tau$ means Mackey's topology on $E_{\tau}^{\prime}$, i.e., the topology of uniform convergence on the convex balanced weakly compact subset of $E$. Indeed, let $f \in \mathscr{F}(\Omega, E)$ and consider the complex valued function, defined in $\Omega \cap C^{n}, z \rightarrow\left\langle e^{\prime}, f(z)\right\rangle$ where $e^{\prime}$ is an arbitrary element in $E^{\prime}$. It is easy to see that the function $\left\langle e^{\prime}, f(z)\right\rangle$ belongs to $\mathscr{F}(\Omega)$. Now let $\alpha$ be arbitrary $2 n$-tuple such that $|\alpha| \leqq m$, and $K$ is a compact set in $\Omega$. Then $\left(D^{\alpha} f(z)\right) e^{-|z| / m}$ stays in a compact subset $\mathcal{K}$ of $E$ as $z$ varies 
in $K \cap \boldsymbol{C}^{n}$, and the closed convex balanced hull $\Gamma(\mathcal{K})$ of $\mathcal{K}$ is weakly compact. If $e^{\prime}$ belongs to the polar of $(1 / \varepsilon) \Gamma(\mathcal{K})$ which is a neighbourhood of zero in $E_{\tau}^{\prime}$, we have

$$
\begin{aligned}
\sup _{z \in K \cap \boldsymbol{C}^{n}}\left|D^{\alpha}\left\langle e^{\prime}, f(z)\right\rangle\right| e^{-|z| / m} & \\
= & \sup _{z \in K \cap \boldsymbol{C}^{n}}\left|\left\langle e^{\prime}, D^{\alpha} f(z)\right\rangle\right| e^{-|z| / m} \leqq \varepsilon .
\end{aligned}
$$

This shows that the mapping $e^{\prime} \rightarrow\left(z \rightarrow\left\langle e^{\prime}, f(z)\right\rangle\right)$ is continuous from $E_{\tau}^{\prime}$ into $\mathscr{I}(\Omega)$.

The proof will be complete if we show that the topology of $\mathscr{I}(\Omega, E)$ is equal to the topology induced by $L_{\varepsilon}\left(E_{\tau}^{\prime}, \mathscr{F}(\Omega)\right)$, the space $L\left(E_{\tau}^{\prime}, \mathscr{I}(\Omega)\right)$ equipped with the topology of uniform convergence on the equicontinuous subset of $E^{\prime}$, since $\varepsilon$ topology on $\mathscr{I}(\Omega) \otimes_{\varepsilon} E$ is the induced topology from $\mathscr{B}_{\varepsilon}\left(E_{\sigma}^{\prime}, \mathscr{I}(\Omega)_{\sigma}^{\prime}\right)$ which is isomorphic to $L_{\varepsilon}\left(E_{\tau}^{\prime}, \mathscr{I}(\Omega)\right)$. (See Proposition 42.2 of F. Treves [15].) Let $U$ be a closed convex balanced neighbourhood of zero in $E, U^{\circ}$ its polar, $K$ a compact set in $\Omega$, and $\alpha \in N^{2 n}$ such that $|\alpha| \leqq m$. Then it is equivalent to say that $\left(D^{\alpha} f(z)\right) e^{-|z| / m} \in U$ for all $z \in K \cap C^{n}$, or to say that $\left|D^{\alpha}\left\langle e^{\prime}, f(z)\right\rangle\right| e^{-|z| / m}$ $\leqq 1$ for all $z \in K \cap \boldsymbol{C}^{n}$ and all $e^{\prime} \in U^{\circ}$. This completes the proof, since a subset of $E^{\prime}$ is equicontinuous if and only if it is contained in the polar of some neighbourhood of zero in $E$.

The following theorem due to P.D.F. Ion and T. Kawai is very useful to go to the vector valued theory from the scalar valued theory.

Theorem 6. 5. Consider the category of Fréchet nuclear spaces where the morphisms are continuous linear maps, and also the category of projectively completed tensor products of Fréchet spaces with metrizable locally convex spaces, where the morphisms are tensor products of some continuous linear map on the Fréchet factor and the identity on the second factor. Let $E$ be a metrizable locally convex space. Then

$$
F \rightarrow F \hat{\otimes} E
$$

and 


$$
L\left(F_{1}, F_{2}\right) \ni u \rightarrow u \hat{\otimes} 1 \in L\left(F_{1} \hat{\otimes} E, F_{2} \hat{\otimes} E\right)
$$

defines a covariant functor from the first mentioned category to the second. This functor is exact.

Proof. See P. D. F. Ion and T. Kawai [4].

Let $\Omega$ be an $\widetilde{\mathcal{O}}_{k, l}$-pseudoconvex domain. From the resolution (4.4) of the sheaf $\widetilde{\mathcal{O}}_{k, l}$ and Theorem 5.9 follows the exact sequence

$$
\mathscr{I}_{(0,0)}(\Omega) \stackrel{\bar{\partial}}{\rightarrow} \mathscr{I}_{(0,1)}(\Omega) \stackrel{\bar{\partial}}{\rightarrow} \cdots \stackrel{\bar{\partial}}{\rightarrow} \mathscr{I}_{(0, n)}(\Omega) \rightarrow 0 .
$$

Therefore from Proposition 2.19, Proposition 6.4 and Theorem 6. 5, we have the exact sequence

$$
\begin{aligned}
0 \rightarrow \widetilde{\mathcal{O}}_{k, l}(\Omega, E) & \rightarrow \mathscr{E}_{(0,0)}(\Omega, E) \stackrel{{ }^{E} \bar{\partial}}{\longrightarrow} \mathscr{E}_{(0,1)}(\Omega, E) \stackrel{{ }^{B} \bar{\partial}}{\longrightarrow} \\
& \stackrel{{ }^{E} \bar{\partial}}{\longrightarrow} \mathscr{E}_{(0, n)}(\Omega, E) \rightarrow 0,
\end{aligned}
$$

where ${ }^{E} \bar{\partial}=\bar{\partial} \hat{\otimes} 1_{E}$ is the Cauchy-Riemann operator for the vector valued functions, and $\widetilde{\mathscr{O}}_{k, l}(\Omega, E)$ is the space of $E$-valued slowly increasing holomorphic functions, that is, the kernel of the Cauchy-Riemann operator. By localizing the above sequence, we have the following soft resolutions of the sheaf ${ }^{E} \widetilde{\mathcal{O}}_{k, l}$ of the $E$-valued slowly increasing functions

$$
0 \rightarrow^{E} \widetilde{\mathcal{O}}_{k, l} \rightarrow^{E} \mathcal{E}_{(0,0)} \stackrel{{ }^{\bar{\partial}} \bar{\longrightarrow}}{\longrightarrow} \mathscr{F}_{(0,1)} \stackrel{{ }^{\bar{\partial}} \bar{\partial}}{\longrightarrow} \ldots \stackrel{E}{\bar{\partial}}^{E} \mathscr{F}_{(0, n)} \rightarrow 0
$$

Theorem 6. 6. If $\Omega$ is an $\widetilde{\mathcal{O}}_{k, l}$-pseudoconvex domain and $E$ is a Fréchet space, then for $p \geqq 1, H^{p}\left(\Omega,{ }^{E} \widetilde{\mathcal{O}}_{k, l}\right)=0$.

Proof. This is a direct consequence of the exact sequence (6.2).

Theorem 6. 7. Let $\Omega$ be an open set in $\boldsymbol{Q}^{k, l}$ satisfying the condition;

$$
\sup _{z \in \Omega \cap C^{n}}\left\{2\left|\operatorname{Im} \underline{z}_{k_{i}}\right|^{2}-\left|\operatorname{Re} z_{i}^{\prime}\right|^{2},\left|\operatorname{Im} \underline{z}_{l_{i}}\right|^{2}\right\} \leqq M<\infty
$$

where $z_{i}^{\prime}=\left(\underline{z}_{k_{i}}, \underline{z}_{l_{i}}\right) \in \boldsymbol{Q}^{k_{i}, l_{i}}$ and $\boldsymbol{Q}^{k, l}=\prod_{i=1}^{j} \boldsymbol{Q}^{k_{i}, l_{i}}$, and $E$ be a Fréchet space. Then $H^{p}\left(\Omega,{ }^{E} \widetilde{\mathcal{O}}_{k, l}\right)=0$ for $p \geqq n$. 
Proof. Theorem 5.11 shows that $H^{n}\left(\Omega, \widetilde{\mathcal{O}}_{k, l}\right)=0$. This shows that the complex $\left(\mathscr{F}_{(0, .)}(\Omega), \bar{\partial}\right)$ is exact at $\mathscr{F}_{(0, n)}(\Omega)$. Therefore it follows from Theorem 6.5 that the complex

$$
0 \rightarrow \mathscr{E}_{(0,0)}(\Omega, E) \stackrel{{ }^{\bar{\partial}}}{\longrightarrow} \ldots \stackrel{E_{\bar{\partial}}}{\longrightarrow} \mathscr{I}_{(0, n)}(\Omega, E) \rightarrow 0 \rightarrow 0 \cdots
$$

is exact at $\mathscr{E}_{(0, n)}(\Omega, E)$. Thus we have $H^{p}\left(\Omega,{ }^{E} \widetilde{\mathcal{O}}_{k, l}\right)=0$ for $p \geqq n$.

Theorem 6. 8. Let $\Omega$ be an open set in $\prod_{i=1}^{j} D^{n_{i}}$ and $E$ be a Fréchet space, then $H_{\Omega}^{p}\left(V,{ }^{E} \widetilde{\mathcal{O}}_{k, l}\right)=0(p \neq n)$ where $V$ is an open set in $\boldsymbol{Q}^{k, l}$ containing $\Omega$ as a relatively closed set.

Proof. It is enough to show $H_{D^{n} \cap V}^{p}\left(V,{ }^{E} \widetilde{\mathcal{O}}_{k, l}\right)=0$ for $p \neq n$ and $V$ an open set in $\boldsymbol{Q}^{k, l}$, where $D^{n}$ is an abbreviation of $\prod_{i=1}^{j} \boldsymbol{D}^{n_{i}}$. By the excision theorem for relative cohomology groups and Theorem 5.3, we may choose $V$ to be an $\widetilde{\mathcal{O}}_{k, l}$-pseudoconvex domain. Let $\Omega=D^{n} \cap V$, then we have the following exact sequence of cohomology groups:

$$
\begin{aligned}
\cdots \rightarrow H_{\Omega}^{p}\left(V,{ }^{E} \widetilde{\mathcal{O}}_{k, l}\right) & \rightarrow H^{p}\left(V,{ }^{E} \widetilde{\mathcal{O}}_{k, l}\right) \rightarrow H^{p}\left(V-\Omega,{ }^{E} \widetilde{\mathcal{O}}_{k, l}\right) \\
& \rightarrow H_{\Omega}^{p+1}\left(V,{ }^{E} \widetilde{\mathcal{O}}_{k, l}\right) \rightarrow \cdots .
\end{aligned}
$$

If $V$ is $\widetilde{\mathcal{O}}_{k, l}$-pseudoconvex, $H^{p}\left(V,{ }^{E} \widetilde{\mathcal{O}}_{k, l}\right)=0$ for $p \geqq 1$ by Theorem 6.6, so that for $p \geqq 2, H_{\Omega}^{p}\left(V,{ }^{E} \widetilde{\mathcal{O}}_{k, l}\right) \cong H^{p-1}\left(V-\Omega,{ }^{E} \widetilde{\mathcal{O}}_{k, l}\right)$. By Theorem 6. 7, we have for $p \geqq n+1, H_{\Omega}^{p}\left(V,{ }^{E} \widetilde{\mathcal{O}}_{k, l}\right)=0$.

For $n-1>p>0$, we have $H^{p}\left(V-\Omega, \widetilde{\mathcal{O}}_{k, l}\right)=0$ from the exact sequence

$$
\begin{aligned}
\rightarrow H_{\Omega}^{p}\left(V, \widetilde{\mathcal{O}}_{k, l}\right) & \rightarrow H^{p}\left(V, \widetilde{\mathcal{O}}_{k, l}\right) \rightarrow H^{p}\left(V-\Omega, \widetilde{\mathcal{O}}_{k, l}\right) \\
& \rightarrow H_{\Omega}^{p+1}\left(V, \widetilde{\mathcal{O}}_{k, l}\right) \rightarrow
\end{aligned}
$$

and the fact that $H_{\Omega}^{p}\left(V, \widetilde{\mathcal{O}}_{k, l}\right)=0$ for $0 \leqq p<n$ (Theorem 5.14). Since $H^{p}\left(V-\Omega, \widetilde{\mathcal{O}}_{k, l}\right)$ is the $p$-th cohomology group of the complex $\left(\mathscr{I}_{(0, .)}(V-\Omega), \bar{\partial}\right)$ and tensoring by $\hat{\otimes} E$ is an exact functor, $H^{p}(V-\Omega$, $\left.{ }^{E} \widetilde{\mathcal{O}}_{k, l}\right)$ vanishes whenever $H^{p}\left(V-\Omega, \widetilde{\mathcal{O}}_{k, l}\right)$ does. Thus for $0<p<n-1$, $H^{p}\left(V-\Omega,{ }^{E} \widetilde{\mathcal{O}}_{k, l}\right)=0$.

Lastly we deal with the sequence

$$
\begin{aligned}
0 \rightarrow H_{\Omega}^{0}\left(V,{ }^{E} \widetilde{\mathcal{O}}_{k, l}\right) & \rightarrow H^{0}\left(V,{ }^{E} \widetilde{\mathcal{O}}_{k, l}\right) \rightarrow H^{0}\left(V-\Omega,{ }^{E} \widetilde{\mathcal{O}}_{k, l}\right) \\
& \rightarrow H_{\Omega}^{1}\left(V,{ }^{E} \widetilde{\mathcal{O}}_{k, l}\right) .
\end{aligned}
$$


Since $H_{\Omega}^{p}\left(V, \widetilde{\mathcal{O}}_{k, l}\right)=0$ for $p=0,1$ if $n \neq 1$, the sequence $0 \rightarrow \widetilde{\mathcal{O}}_{k, l}(V)$ $\rightarrow \widetilde{\mathcal{O}}_{k, l}(V-\Omega) \rightarrow 0$ is exact. Since $\widetilde{\mathcal{O}}_{k, l}(W)$ for an open set $W$ in $\boldsymbol{Q}^{k, l}$ is a closed subspace of a nuclear Fréchet space $\mathscr{F}(W), \widetilde{\mathcal{O}}_{k, l}(W)$ is also a nuclear Fréchet space. By Theorem 6.5 we have the exact sequence

$$
0 \rightarrow \widetilde{\mathcal{O}}_{k, l}(V) \hat{\otimes} E \rightarrow \widetilde{\mathcal{O}}_{k, l}(V-\Omega) \hat{\otimes} E \rightarrow 0 .
$$

Since $\operatorname{ker}(\bar{\partial} \hat{\otimes} 1)=(\operatorname{ker} \bar{\partial}) \hat{\otimes} E$, we have

$$
\widetilde{\mathcal{O}}_{k, l}(W) \hat{\otimes} E=\widetilde{\mathcal{O}}_{k, l}(W, E) .
$$

Thus we have the exact sequence

$$
0 \rightarrow \widetilde{\mathcal{O}}_{k, l}(V, E) \rightarrow \widetilde{\mathcal{O}}_{k, l}(V-\Omega, E) \rightarrow 0,
$$

that is, $H_{a}^{p}\left(V,{ }^{E} \widetilde{\mathcal{O}}_{k, l}\right)=0$ for $p=0,1$. The proof is complete if $n \neq 1$. The case $n=1$ follows at once from Theorem 6.6 and Theorem 6.7.

Now we define the space of Fourier hyperfunctions as the only nonvanishing relative cohomology group.

Definition 6.9. Let $E$ be a Fréchet space. The space ${ }^{E} \mathscr{R}_{k, l}(\Omega)$ of $E$-valued Fourier hyperfunctions of $(k, l)$ type on an open set $\Omega$ in $\prod_{i=1}^{j} \boldsymbol{D}^{n_{i}}$ is defined to be ${ }^{E} \mathcal{R}_{k, l}(\Omega)=H_{\Omega}^{n}\left(V,{ }^{E} \widetilde{\mathcal{O}}_{k, l}\right)$ where $V$ is an open set in $\boldsymbol{Q}^{k, l}$ containing $\Omega$ as a closed subset.

Proposition 6. 10. ${ }^{E} \mathscr{R}_{k, l}(\Omega) \cong H_{\bar{\Omega}}^{n}\left(V,{ }^{E} \widetilde{\mathcal{O}}_{k, l}\right) / H_{\partial \Omega}^{n}\left(V,{ }^{E} \widetilde{\mathcal{O}}_{k, l}\right)$ where $\bar{\Omega}$ is the closure of $\Omega$ in $\boldsymbol{Q}^{k, l}$ and $\partial \Omega$ is boundary of $\Omega$, i.e., $\bar{\Omega}-\Omega$.

Proof. Consider the following exact sequence:

$$
\begin{gathered}
\rightarrow H_{\Omega}^{n-1}\left(V-\partial \Omega,{ }^{E} \widetilde{\mathcal{O}}_{k, l}\right) \rightarrow H_{\partial \Omega}^{n}\left(V,{ }^{E} \widetilde{\mathcal{O}}_{k, l}\right) \rightarrow H_{\bar{\Omega}}^{n}\left(V,{ }^{E} \widetilde{\mathcal{O}}_{k, l}\right) \\
\rightarrow H_{\Omega}^{n}\left(V-\partial \Omega,{ }^{E} \widetilde{\mathcal{O}}_{k, l}\right) \rightarrow H_{\partial \Omega}^{n+1}\left(V,{ }^{E} \widetilde{\mathcal{O}}_{k, l}\right) \rightarrow,
\end{gathered}
$$

where $V$ is an open neighbourhood of $\bar{\Omega}$ in $\boldsymbol{Q}^{k, l}$. By Theorem 6.8, we have $H_{\Omega}^{n-1}\left(V-\partial \Omega,{ }^{E} \widetilde{\mathcal{O}}_{k, l}\right)=0$, and from the exact sequence

$$
\rightarrow H^{n}\left(V-\partial \Omega,{ }^{E} \widetilde{\mathcal{O}}_{k, l}\right) \rightarrow H_{\partial \Omega}^{n+1}\left(V,{ }^{E} \widetilde{\mathcal{O}}_{k, l}\right) \rightarrow H^{n+1}\left(V,{ }^{E} \widetilde{\mathcal{O}}_{k, l}\right) \rightarrow,
$$

and Theorem 6. 7, we have $H_{\partial \Omega}^{n+1}\left(V,{ }^{E} \widetilde{\mathcal{O}}_{k, l}\right)=0$. Therefore we have the required isomorphism. 
Theorem 6. 11. The presheaf $\left\{{ }^{E} \mathcal{R}_{k, l}(\Omega)\right\}$ constitutes a flabby sheaf ${ }^{E} \mathscr{R}_{k, l}$ over $\prod_{i=1}^{j} \boldsymbol{D}^{n_{i}}$, whose restriction to $\boldsymbol{R}^{n}$ coincides with the sheaf of E-valued hyperfunctions over $\boldsymbol{R}^{n}$.

Proof. The flabbiness of ${ }^{E} \mathscr{R}_{k, l}$ is the direct consequence of Theorem 6. 8. Since the restriction of the sheaf ${ }^{E} \widetilde{\mathcal{O}}_{k, l}$ to $C^{n}$ coincides with the sheaf of $E$-valued holomorphic functions ${ }^{E} \mathcal{O},{ }^{E} \mathcal{R}_{k, l} \mid \boldsymbol{R}^{n}$ coincides with the sheaf of $E$-valued hyperfunctions defined in P. D. F. Ion and T. Kawai [4].

We represent the $E$-valued Fourier hyperfunctions as boundary values of $E$-valued slowly increasing holomorphic functions. From Theorem 5.3 there exists an $\widetilde{\mathcal{O}}_{k, l}$-pseudoconvex neighbourhood $V$ of $\Omega$ such that $\left(\prod_{i=1}^{j} D^{n_{i}}\right) \cap V=\Omega$. Let $V_{j}=\left\{z \in V ; \operatorname{Im} z_{j} \neq 0\right\}, j=1,2, \cdots, n$, then $V_{j}$ is $\widetilde{\mathcal{O}}_{k, l}$-pseudoconvex. In fact, let $\theta(z)$ be a plurisubharmonic function which satisfies the conditions of Definition 5.1 for the $\widetilde{\mathcal{O}}_{k, l}$-pseudoconvex domain $V$, then the plurisubharmonic function $p_{j}(z)+\theta(z)$ satisfies the conditions of Definition 5.1 for $V_{j}$, where $p_{j}(z)=\log \left(1 /\left|\operatorname{Im} z_{j}\right|^{2}\right)$ or $p_{j}(z)$ $=\log \left(\left(1+\left|z_{i}^{\prime}\right|^{2}\right) /\left|\operatorname{Im} z_{j}\right|^{2}\right)$ if $z_{j}$ is a component of some $\underline{z}_{l_{i}}$ or $\underline{z}_{k_{i}}$ respectively, where $z_{i}^{\prime}=\left(\underline{z}_{k_{i}}, \underline{z}_{l_{i}}\right) \in \boldsymbol{Q}^{k_{i}, l_{i}}$ and $\boldsymbol{Q}^{k_{1} l}=\prod_{i=1}^{j} \boldsymbol{Q}^{k_{i}, l_{i}}$. Let $V_{0}=V, \subset V^{\prime}=\left\{V_{1}\right.$, $\left.\cdots, V_{n}\right\}, C V=\left\{V_{0}\right\} \cup C V^{\prime}$ then $\left(C, C V^{\prime}\right)$ is a $\widetilde{\mathcal{O}}_{k, l}$-pseudoconvex relative covering of $(V, V-\Omega)$. Since the finite intersection of $\widetilde{\mathcal{O}}_{k, l}$-pseudoconvex domains is also $\widetilde{\mathcal{O}}_{k, l}$-pseudoconvex, we have $H^{p}\left(V_{j_{l}} \cap \cdots \cap V_{j_{k}},{ }^{E} \widetilde{\mathcal{O}}_{k, l}\right)$ $=0(p \geqq 1)$ for any $j_{1}, \cdots, j_{k}$ from Theorem 6.6. Then we have the following isomorphism from a theorem of Leray,

$$
{ }^{E} \mathcal{R}_{k, l}(\Omega) \cong H^{n}\left(C, C V^{\prime},{ }^{E} \widetilde{\mathcal{O}}_{k, l}\right)={ }^{E} \widetilde{\mathcal{O}}_{k, l}(V \# \Omega) / \sum_{j}{ }^{E} \widetilde{\mathcal{O}}_{k, l}\left(\widehat{V}_{j}\right),
$$

where $V \# \Omega=\left\{z \in V ; \operatorname{Im} z_{j} \neq 0\right.$, for all $\left.j\right\}$ and $\widehat{V}_{j}=\left\{z \in V ; \operatorname{Im} z_{k} \neq 0\right.$, if $k \neq j\}$. Thus the element $f$ of ${ }^{E} \widetilde{\mathcal{O}}_{k, l}(V \# \Omega)$ defines the $E$-valued Fourier hyperfunction $[f]$ and we call $f$ the defining function of $[f]$. This function $f(z)$ can be considered as $2^{n}$ tuple of $E$-valued slowly increasing holomorphic functions $f_{\sigma}(z)$ defined in $V \cap\left(\boldsymbol{R}^{n}+i \Gamma_{\sigma}\right)$ where $\Gamma_{\sigma}=\left\{y \in \boldsymbol{R}^{n}\right.$; $\left.\sigma_{j} y_{j}>0\right\}$ and $\sigma: j \rightarrow \sigma_{j}$ is a mapping from $\{1, \cdots, n\}$ to $\{1,-1\}$. Intuitively speaking, the hyperfunction $[f]$ is the sum of boundary values

$$
[f](x)=\sum_{\sigma}\left(\prod_{j=1}^{n} \sigma_{j}\right) f\left(x+i \Gamma_{\sigma} 0\right) .
$$




\section{References}

[1] Gelfand, I. M. and Shilov, G. E., Generalized Functions, 2, Academic Press, New York-London, 1968.

[2] Hörmander, L., Complex Analysis in Several Variables, North-Holland, AmsterdamLondon, 1973.

[3] Inoue, A., Unbounded generalization of standard von Neumann algebras, Report on Math. Phys., 13 (1978), 25-35.

[4] Ion, P. D. F. and Kawai, T., Theory of vector-valued hyperfunctions, Publ. RIMS, Kyoto Univ., 11 (1975), 1-19.

[5] Ito, Y. and Nagamachi, S., On the theory of vector valued Fourier hyperfunctions, Jour. Math. Tokushima Univ., 9 (1975), 1-33.

[6] Kawai, T., On the theory of Fourier hyperfunctions and its applications to partial differential equations with constant coefficients, J. Fac. Sci. Univ. Tokyo, Sect. IA, 17 (1970), 467-517.

[7] Komatsu, H., Projective and injective limits of weakly compact sequences of locally convex spaces, J. Math. Soc. Japan, 19 (1967), 366-383.

[8] Mityagin, B. S., Nuclearity and other properties of spaces of type S, Trudy Moskov. Mat. Obšč, 9 (1960), 317-328 (in Russian).

[9] Nagamachi, S. and Mugibayashi, N., Hyperfunction quantum field theory, Commun. math. Phys., 46 (1976), 119-134.

[10] - Hyperfunction quantum field theory II, Euclidnean Green's functions, ibid., 49 (1976), 257-275.

[11] - Quantum field theory in terms of Fourier hyperfunctions, Publ. RIMS, Kyoto Univ., 12 Suppl., (1977), 309-341.

[12] Pietsch, A., Nuclear Locally Convex Spaces, Springer, Berlin-Heidelberg-New York, 1972.

[13] Saburi, Y., On Fourier hyperfunctions, Master thesis at Sophia Univ., 1978 (in Japanese).

[14] - Vanishing theorems of cohomology groups with coefficients in sheaves of holomorphic functions with bounds, Proc. Japan Acad, 54A (1978), 274-378.

[15] Treves, F., Topological Vector Spaces, Distributions and Kernels, Academic Press, New York-London, 1967. 
